ARTICLE

https://doi.org/10.1038/s41467-019-12355-7

\title{
Catalytically inactive Dnmt3b rescues mouse embryonic development by accessory and repressive functions
}

Pawel Nowialis1,2,10, Katarina Lopusna1,2,10, Jana Opavska1,2, Staci L. Haney ${ }^{3}$, Ajay Abraham (1) 1,2, Peike Sheng ${ }^{4}$, Alberto Riva (1) ${ }^{5}$, Amarnath Natarajan (1) 6 , Olga Guryanova7, Melanie Simpson ${ }^{8}$, Ryan Hlady (D) ${ }^{9}$, Mingyi Xie $^{2,4}$ \& Rene Opavsky ${ }^{1,2 \star}$

DNA methylation regulates gene expression in a variety of processes, including mouse embryonic development. Four catalytically active enzymes function in mice as DNA methyltransferases (Dnmts) and as transcriptional regulators. Inactivation of Dnmt3b results in mouse embryonic lethality, but which activities are involved is unclear. Here we show that catalytically inactive Dnmt3b restores a majority of methylation and expression changes deregulated in the absence of Dnmt3b, and as a result, mice survive embryonic development. Thus, Dnmt3b functions as an accessory cofactor supporting catalytic activities performed by other Dnmts. We further demonstrate that Dnmt3b is linked to a control of major developmental pathways, including Wnt and hedgehog signaling. Dnmt3b directly represses Wnt9b whose aberrant up-regulation contributes to embryonic lethality of Dnmt3b knockout embryos. Our results highlight that Dnmt3b is a multifaceted protein that serves as an enzyme, an accessory factor for other methyltransferases, and as a transcriptional repressor in mouse embryogenesis.

\footnotetext{
${ }^{1}$ Department of Anatomy and Cell Biology, University of Florida College of Medicine, 2033 Mowry Rd, CGRC 258, Gainesville, FL 32610, USA. ${ }^{2}$ UF Health Cancer Center, University of Florida, Gainesville, FL 32610, USA. ${ }^{3}$ Department of Internal Medicine, University of Nebraska Medical Center, 985950 Nebraska Medical Center, Omaha, NE 68198, USA. ${ }^{4}$ Department of Biochemistry and Molecular Biology, University of Florida College of Medicine, 2033 Mowry Rd, CGRC 263, Gainesville, FL 32610, USA. ${ }^{5}$ ICBR Bioinformatics, Cancer and Genetics Research Complex, University of Florida, P.O. Box 103622, Gainesville, FL 32610, USA. ${ }^{6}$ University of Nebraska Medical Center, The Eppley Institute for Research in Cancer and Allied Diseases, Fred \& Pamela Buffett Cancer Center, 986805 Nebraska Medical Center, Omaha, NE 68198, USA. ${ }^{7}$ Department of Pharmacology and Therapeutics, University of Florida College of Medicine, 2033 Mowry Rd, CGRC 260, Gainesville, FL 32610, USA. ${ }^{8}$ Molecular and Structural Biochemistry, NC State University, Raleigh, NC 27695, USA. ${ }^{9}$ Department of Molecular Pharmacology and Experimental Therapeutics, Mayo Clinic, Rochester, MN 55901, USA. ${ }^{10}$ These authors contributed equally: Pawel Nowialis, Katarina Lopusna. *email: ropavsky@ufl.edu
} 
D NA methylation of $\mathrm{CpG}$ dinucleotides is a covalent modification involved in regulation of gene expression in mammalian cells. It has suppressive effects on gene transcription, especially in CpG islands (CGIs) of gene promoters or in enhancers where it is often associated with repressive histone modifications such as $\mathrm{H} 3 \mathrm{~K} 9 \mathrm{me} 3$ and H3K27me3 (refs. ${ }^{1,2}$ ). Methylation can also enhance transcription by promoting more efficient binding of transcription factors to their recognition sites ${ }^{3,4}$ and by restricting the activation of alternative promoters within gene bodies 4 . Thus, effects of DNA methylation on gene expression are complex and context-dependent.

The family of DNA methyltransferases (Dnmts) consists of four catalytically active enzymes (Dnmt1, Dnmt3a, Dnmt3b, and Dnmt3c) and one catalytically inactive cofactor (Dnmt3L) in mice. All Dnmts participate in generation and maintenance of methylation highlighting complexity of this epigenetic phenomenon $^{5-7}$. Dnmt3L is an accessory protein that lacks catalytic activity (CA) but is critical for induction of de novo methylation by linking Dnmt3a and Dnmt3b to chromatin through unmethylated H3 lysine 4 (ref. ${ }^{8}$ ). Dnmt3c suppresses transposon activity specifically in male germ cells ${ }^{9}$. Apart from CA, Dnmts suppress transcription through repression by association with HDACs, MBDs, Rb, and other Dnmts ${ }^{10}$. In addition, Dnmts can associate with areas of active gene transcription. DNMT3B selectively binds to the bodies of actively transcribed genes through the interaction of its PWWP domain with histone $\mathrm{H} 3$ trimethylated at lysine 36 , and plays a role in their preferential methylation in embryonic stem cells ${ }^{11}$.

DNA methylation plays a major role in development from conception. After fertilization, methylation is erased at the blastocyst stage, and then re-established between 4.5 and 6.5 days post coitum in murine epiblast cells by the combined action of DNMT3A and DNMT3B. Newly generated methylation patterns form a basis for tissue-specific expression, and are utilized in cell differentiation and organogenesis later during embryogenesis ${ }^{12,13}$. Consistently with the importance of methylation, inactivation of Dnmt1 or Dnmt3b is embryonically lethal at E9.5 and $\sim$ E11.5-15.5, respectively ${ }^{5,6,14}$. Dnmt3 $a^{-/-}$mice die shortly after the birth ${ }^{5}$. The contribution of Dnmt3b to genome methylation is greater than that of Dnmt3a and consists of specific methylation of sequences associated with developmental and imprinted genes, and X chromosome inactivation ${ }^{13}$.

Because of numerous activities including catalytic and repressive functions, as well as expression of numerous DNMT3b isoforms, it is difficult to understand which physiological processes solely depend on Dnmt3b's CA ${ }^{15,16}$. To understand contribution of Dnmt3b's CA activity to embryogenesis, we generated and analyzed mice expressing catalytically inactive Dnmt3b allele $\left(D n m t 3 b^{C I}\right)$ from the endogenous locus. Dnmt3b $b^{C I / C I}$ survive embryonic development because catalytically inactive Dnmt3b restores a majority of molecular changes observed in $D n m t 3 b^{-/-}$ mice. We further show that aberrant activation of Wnt signaling contributes to lethality of Dnmt3b-/- embryos and that Dnmt3b inhibits this pathway by repressing Wnt9b. Thus, Dnmt3b functions as an accessory factor to other Dnmts and as a transcriptional repressor in mouse embryogenesis.

\section{Results}

Catalytic activity of Dnmt3b is dispensable for embryogenesis. To address whether Dnmt3b's CA is important for mouse embryogenesis, we sought to generate Dnmt3b allele lacking CA. A replacement of cysteine at position 657 by a serine or threonine in mouse Dnmt3b coding sequence (CDS) has been shown to abolish CA without compromising other functions ${ }^{17,18}$. Similarly, double amino acid (aa) substitution P705V/C706D in the CDS of
Dnmt3a inactivates its $\mathrm{CA}^{19}$. Therefore, we considered substitution Dnmt3b $b^{\mathrm{P} 656, \mathrm{C} 657}$ (catalytic site corresponding to Dnmt3a ${ }^{\mathrm{P} 705, \mathrm{C} 706}$ ) for valine and aspartate, respectively. Such substitution is predicted to disable first two steps (nucleophilic addition and methyl transfer) of the three-step process required for CA, thus rendering Dnmt3b $\mathrm{b}^{\mathrm{P} 656 \mathrm{~V} / \mathrm{C} 657 \mathrm{D}}$ protein catalytically inactive (CI; referred to here as Dnmt3bCI) (Fig. 1a, Supplementary Fig. 1).

To test CA, we expressed both wild-type (Dnmt3b $\mathrm{b}^{\mathrm{WT}}$ ) and Dnmt3b $b^{C I}$ in Dnmt $3 a^{-/-} ;$Dnmt $3 b^{-/-}$mouse lymphoma cell line $^{20}$ and analyzed global methylation by reduced representation bisulfite sequencing (RRBS). In this assay, the ability of Dnmt3b to induce differentially methylated regions (DMRs; defined as $\geq 30 \%$ methylation change in three consecutive cytosines in $\leq 100$ bp) was severely diminished ( 10 -fold) relative to Dnmt $3 b^{\text {WT }}$ (Supplementary Fig. 2). Residual activity of Dnmt3b ${ }^{\mathrm{CI}}$ may stem from complexing with intracellular Dnmts, such as Dnmt1. Although we cannot rule out that $\mathrm{Dnmt}_{3 \mathrm{~b}} \mathrm{CI}$ retains some residual CA activity, our bioinformatics and functional assays strongly suggest that this protein lacks CA.

To generate mice expressing Dnmt $3 b^{C I}$ protein from the endogenous locus, we utilized CRISPR/Cas9 homology-directed repair to target exon 19 of Dnmt3b by co-injection of mRNA, sgRNA, and donor oligo into FVB mouse zygotes (Fig. 1a). A proper targeting was confirmed by PCR-based genotyping followed by sequencing. Dnmt $3 b^{+/ C I}$ founders were born without any detectable phenotypes with long lifespan. Subsequent analysis showed no phenotypic differences; therefore, we further do not distinguish between founder-derived offspring.

Interbreeding of $D n m t 3 b^{+/ C I}$ mice yielded Dnmt $3 b^{C I / C I}$ mice that were surprisingly alive at weaning age although the genetic ratios were slightly lower than expected (Fig. 1b-d). Interestingly, Dnmt $3 b^{C I / C I}$ mice were $\sim 20 \%$ smaller than their $D n m t 3 b^{+/+}$ littermates at weaning and such differences persisted throughout their lives (Fig. 1e). Both male and female Dnmt3b $b^{C I / C I}$ mice are fertile and have normal lifespan.

Dnmt $3 b^{C I}$ levels were indistinguishable from Dnmt3b ${ }^{W T}$ levels in adult tissues suggesting that an introduction of aa substitutions did not adversely affect regulation of Dnmt3b expression or protein stability (Fig. 1f).

Because embryonic lethality of Dnmt $3 b^{-/-}$mice at E11.5-15.5 was previously addressed using unrelated mouse strains ${ }^{5,6}$, we next asked whether Dnmt $3 b^{-/-}$embryos derived from the FVB genetic background can, like $D n m t 3 b^{C I / C I}$ mice, survive embryogenesis. We found that Dnmt $3 b^{-/-}$embryos are underrepresented at E11.5 and E12.5, suggesting embryonic lethality in this mouse strain (Fig. 1g). Morphological analysis revealed heart defects, including enlarged pericard with edema, and hemorrhage in Dnmt $3 b^{-/-}$but not in Dnmt $3 b^{C I / C I}$ embryos (Fig. 1h, Supplementary Fig. 3).

Like in adult tissues, Dnmt3b $\mathrm{b}^{\mathrm{CI}}$ protein levels in $\mathrm{Dnm} t 3 b^{\mathrm{CI} / \mathrm{CI}}$ embryos were comparable to WT counterparts. Dnmtl and Dnmt3a were also unchanged, suggesting that the observed rescue of embryogenesis is not due to compensation by these proteins (Fig. 1i). Interestingly, Dnmt3L was up-regulated in Dnmt3b-/- but not in Dnmt3bCI/CI embryos suggesting potential functional compensation for the loss of Dnmt3b (Fig. 1i). Global level of 5methylcytosine in $D n m t 3 b^{C I / C I}$ was reduced relative to Dnmt3b $b^{+/+}$ but not to Dnmt3b-/- E11.5 embryos (Fig. 1j). Furthermore, methylation of two Dnmt3b target genes, $D d x 4$ (ref. ${ }^{13}$ ) and Ment ${ }^{21}$, was similarly reduced in both Dnmt3 $b^{-/-}$and Dnmt3b $b^{C I / C I}$ E11.5 embryos (Supplementary Figs. 4 and 5). These data further suggest that Dnmt3bCI lacks CA in vivo.

To determine if the rescue of embryogenesis is limited to the FVB strain, we generated independent mouse lines through targeting of $\mathrm{C} 57 \mathrm{BL} / 6$ strain zygotes as outlined in Fig. 1a. 
a

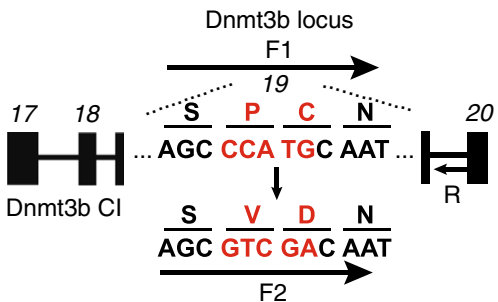

b

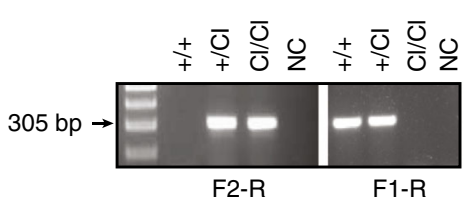

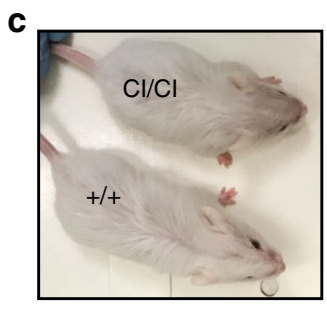

d

\begin{tabular}{|c|c|c|}
\hline FVB & Observed & Expected \\
\hline$+/+$ & $10925 \%$ & $10925 \%$ \\
\hline$+/ \mathrm{Cl}$ & $23053 \%$ & $21850 \%$ \\
\hline $\mathrm{Cl} / \mathrm{Cl}$ & $97 \quad 22 \%$ & $10925 \%$ \\
\hline Total & 436 & \\
\hline
\end{tabular}

e

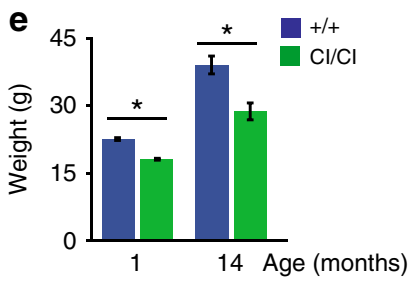

f

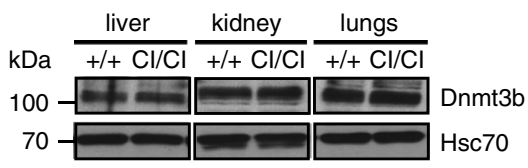

g

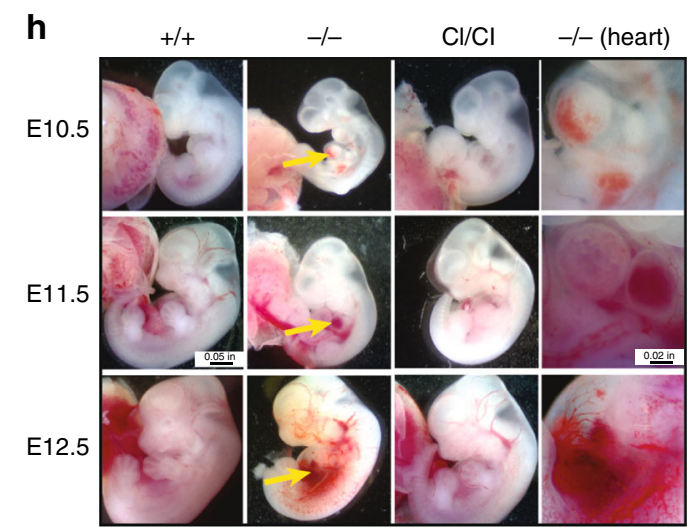

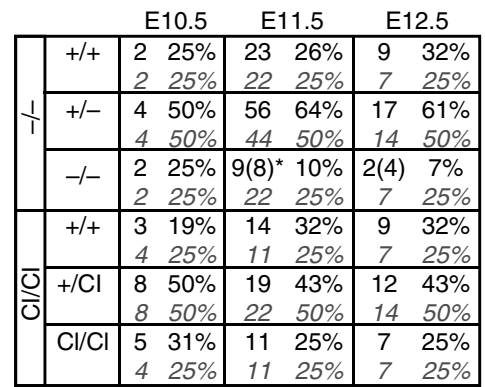
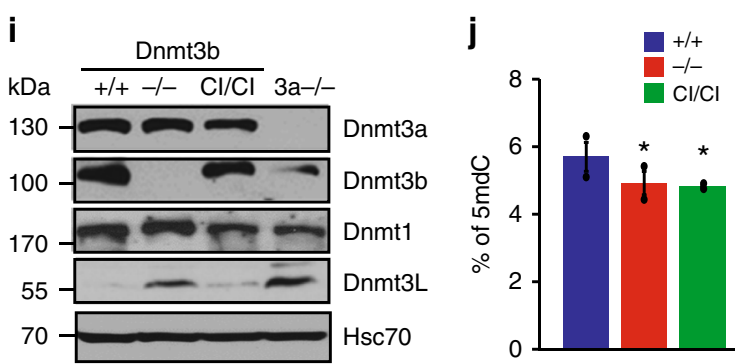

k

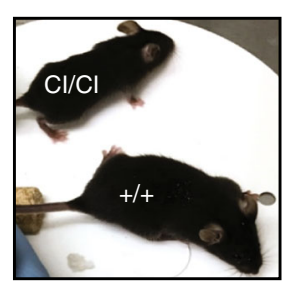

I

\begin{tabular}{|c|cc|cc|}
\hline C57BL/6 & \multicolumn{2}{|c|}{ Observed } & \multicolumn{2}{|c|}{ Expected } \\
\hline$+/+$ & 28 & $29 \%$ & 24 & $25 \%$ \\
\hline$+/ \mathrm{Cl}$ & 47 & $49 \%$ & 48 & $50 \%$ \\
\hline $\mathrm{Cl} / \mathrm{Cl}$ & 21 & $22 \%$ & 24 & $25 \%$ \\
\hline Total & 96 & & & \\
\hline
\end{tabular}

Fig. 1 Catalytically inactive Dnmt3b is sufficient for normal mouse embryogenesis. a The schematic illustrates exons 17-20 (black boxes) of the mouse Dnmt3b, a portion with nucleotide and amino acid sequences in exon 19 encoding wild type (top) and sequence encoding amino acid substitution: PC to VD inactivating catalytic activity that was used to generate $\mathrm{Dnmt} 3 \mathrm{~b}^{\mathrm{Cl}}$ allele (bottom). Position of genotyping primers is indicated by arrows. F1-R detects wild-type sequence; F2-R detects mutated sequence. b PCR genotyping assay performed on DNA isolated from Dnmt $3 b^{+/+}(+/+), \mathrm{Dnmt} 3 \mathrm{~b}^{+/ \mathrm{Cl}}(+/ \mathrm{Cl})$ and $\mathrm{Dnmt} 3 \mathrm{~b}^{\mathrm{Cl} / \mathrm{Cl}}$ $(\mathrm{Cl} / \mathrm{Cl})$ mice. F1-R and F2-R represents primer pairs detecting wild type and $\mathrm{Cl}$ allele, respectively. $\mathrm{NC}$, no template control. c Micrograph of 6-week-old $+/+$ and $\mathrm{Cl} / \mathrm{Cl}$ mice. $\mathbf{d}$ Genotypic analysis of offspring derived from Dnmt3b $b^{+/ C l}$ intercrosses ( $F V B$ strain). e The weight of Dnmt $3 b^{+/+}(+/+)$and $D n m t 3 b^{C l} / \mathrm{Cl}(\mathrm{Cl} /$ $\mathrm{Cl}$ ) mice measured at $1(n=16)$ and 14 months $(n=4)$. Data are presented as mean $\pm S E M,{ }^{*} p<0.001$ (two-tailed Student's $t$-test). $\mathbf{f}$ Immunoblot showing Dnmt3b protein levels in liver, kidney, and lungs of 8 weeks old $+/+$ and $\mathrm{Cl} / \mathrm{Cl}$ mice. Hsc70 served as a loading control. $\mathbf{g}$ Genotypic analysis of embryos isolated from intercrosses of Dnmt3b+/- and Dnmt3b+/Cl mice at E10.5, E11.5, and E12.5. Bold font indicates observed numbers and gray italic indicates expected number. Numbers in brackets denote dead embryos. ${ }^{\star} p<0.01$ ( $\chi^{2}$-test). h Gross morphology of Dnmt3b+/+, Dnmt3b-/- and Dnmt3b $\mathrm{Cl} / \mathrm{Cl}$ E10.5, E11.5, and E12.5 embryos (magnification $\times 8$ ). Arrows point to hemorrhages and pericard edema of Dnmt3b-/- mice. Higher-magnification $(\times 40)$ images of hearts are shown in the right panel. i Immunoblot showing Dnmt3a, Dnmt3b, Dnmt1, Dnmt3L, and Hsc70 (loading control) protein levels in E11.5 Dnmt3b $+/+$ $(+/+)$, Dnmt3b $b^{-/-}(-/-), D n m t 3 b^{C l} / C l(C l / C l)$, and Dnmt3a-/- $\left(3 a^{-/-}\right)$whole embryo lysates. This labeling of genetic groups is used in all subsequent figures unless noted otherwise. j Percentage of methylated cytosines in genomic DNA in Dnmt $3 b^{+/+}(+/+), \mathrm{Dnmt}^{+} b^{-/-}\left({ }^{-/-}\right), \mathrm{Dnmt} 3 b^{\mathrm{Cl} / \mathrm{Cl}}(\mathrm{Cl} / \mathrm{Cl}) \mathrm{E} 11.5$ embryos $(n=2)$ as determined by mass spectrometry. Data are presented as mean \pm SEM. ${ }^{*} p<0.05$ (two-tailed Student's $t$-test). k Micrograph of 6 -weekold $+/+$ and $\mathrm{Cl} / \mathrm{Cl}$ mice (C57BL/6 strain). I Genotypic analysis of offspring derived from intercrosses of Dnmt $3 b^{+/ C l}$ mice (C57BL/6 background). Source data are provided as a Source Data file 
Interbreeding of $D n m t 3 b^{+/ C I}$ mice yielded viable $D n m t 3 b^{C I / C I}$ pups (Fig. 1k, l), demonstrating that the rescue of embryogenesis by Dnmt3b $\mathrm{b}^{\mathrm{CI}}$ is not strain-specific.

Altogether, these results demonstrate that CA of Dnmt3b is, surprisingly, dispensable for mouse embryogenesis.

Accessory activity of Dnmt3b restores global methylation. To address the effects of Dnmt3b deficiency, we next analyzed global DNA methylation patterns in Dnmt $3 b^{+/+}, D n m t 3 b^{-/-}$, and $D n m t 3 b^{C I / C I}$ embryos at E11.5. RRBS yielded methylation information on more than million CGs with coverage $\geq 15 \times$, out of which $>8 \times 10^{5}$ CGs present in all samples were used for the further analysis (Supplementary Fig. 6). Most of the differentially methylated cytosines (DMCs) in Dnmt $3 b^{-/-}$embryos were hypomethylated relative to WT counterparts, but these changes were substantially reduced in Dnmt3b $b^{C I / C I}$ embryos (Fig. 2a, b). Further analysis revealed $>4000$ hypomethylated and no hypermethylated DMRs in Dnmt $3 b^{-1-}$ embryos (Fig. 2c). In contrast, only 315 hypomethylated DMRs were identified in E11.5 Dnmt $3 b^{C I / C I}$ embryos, most of which overlapped with DMRs found in Dnmt3b-/- embryos (Fig. 2c). Thus, the presence of Dnmt $3 b^{C I}$ resulted in restoration of DNA methylation of $\sim 95 \%$ (4133/4338) of DMRs that was lost in Dnmt3b-/- embryos. Such effect was observed across the genome and as a result methylation of $D n m t 3 b^{C I / C I}$ embryos clusters apart from $D n m t 3 b^{-/}$and close to WT (Fig. 2d-g, Supplementary Fig. 7, Supplementary Data 1).

Dnmt3b is dispensable for methylation of germline imprinted control regions (gICRs) but involved in methylation of secondary somatic ICRs (sICRs) ${ }^{13,22-24}$. To gain further insight into Dnmt3b functions, we next analyzed methylation of 22 gICRs and 9 sICRs $^{22-24}$. As expected, Dnmt3b was dispensable for methylation of majority of gICRs including Impact (Fig. 2h, Supplementary Data 2). However, we observed substantial hypomethylation in Gnas1a, Dlk1-Gtl2, 6330408a02Rik, and AK008011 gICRs in E11.5 Dnmt3b ${ }^{-/-}$embryos. Methylation was restored to WT levels only for Gnas1a and Dlk1-Gtl2 in $D n m t 3 b^{C I / C I}$ embryos, suggesting that CA of Dnmt3b is critical for 6330408a02Rik and AK008011 loci (Fig. 2h, Supplementary Fig. 8, Supplementary Data 2).

Similarly, we also observed hypomethylation of 7/9 sICRs (Mkrn3, Nesp, Cdkn1c, Gtl2, H19, Peg12, Igf2r) in Dnmt3b $b^{-1-}$ embryos that was restored only for H19, Nesp, Gtl2, and Igf $2 r$ in $D n m t 3 b^{C I / C I}$ embryo (Fig. 2i, Supplementary Fig. 8). The remaining sICRs (diffuse DMRs- Gtl2-Mirg and Eif2c2) were not targeted by Dnmt3b, since they were not changed in methylation (Supplementary Data 2).

Altogether, our analysis revealed that a vast majority of methylation changes observed in Dnmt $3 b^{-/-}$embryos is restored in Dnmt3b $b^{C I / C I}$ to WT levels consistently with the successful rescue of embryogenesis. Thus, Dnmt3b plays a role as accessory factor for DNA methylation independent of its intrinsic catalytic activity.

Activation of demethylated promoters lacking histone marks. To analyze effects of Dnmt3b, we performed global gene expression RNA profiling from E11.5 WT, Dnmt $3 b^{-/-}$, and Dnmt $3 b^{C I / C I}$ embryos using RNA-sequencing. In total, 253 upregulated and 819 downregulated genes $(\mathrm{FC} \geq 2)$ were identified in Dnmt3 ${ }^{-/-}$embryos relative to WT controls (Fig. 3a). The most up-regulated genes were related to germ cells specific expression (e.g. D1Pas1, Sohlh2) while most downregulated genes included crystallins (e.g. Cryga, Cryba4) and matrilins (e.g. Matn1, Matn4). In addition, multiple poorly characterized protein coding genes such as Gm43302, Gm43638, Gm37034 and non-coding RNA genes (9330158H04Rik; Gm22009) were also deregulated (Supplementary Data 3).

Like for DNA methylation, a vast majority of gene expression was rescued to the WT levels with only a subset of genes deregulated in Dnmt3bCI/CI embryos (Fig. 3a). As a result, expression profiles of WT and Dnmt3bCI/CI embryos clustered closer and apart of Dnmt3b-/- (Fig. 3b, Supplementary Data 3).

We next analyzed association between DNA methylation and gene expression in various genomic elements. This analysis revealed that hypomethylation of $\sim 9 \%$ (34/401) long promoters was associated with increased gene expression in Dnmt $3 b^{-/}$embryos (Fig. 3c). Other elements, including gene bodies and enhancers, correlated with changes in methylation to a lower extent (Supplementary Fig. 9). Expression of few genes remained elevated in Dnmt3b $b^{C I / C I}$ embryos but this did not interfere with normal embryogenesis (Fig. 3a).

We next focused our analysis on the relationship between gene expression and hypomethylation in gene promoters. Out of 401 promoters methylated in WT, only 34 responded to hypomethylation by increased expression in E11.5 Dnmt $3 b^{-/-}$embryos. We therefore asked whether other epigenetic changes already present in E11.5 WT embryos could predetermine increased expression upon hypomethylation in Dnmt $3 b^{-/-}$embryos. To address this, we utilized genome-wide profiles of eight histone modifications generated from E11.5 WT embryos available at ENCODE (Bing Ren, UCSD lab, ENCSR231EPI, ENCSR215ZYV) ${ }^{25}$. By combining information on gene expression, DNA methylation, histone modifications, and chromatin accessibility, we identified three groups of genes with variable effects on expression in response to methylation loss.

The first group (Group 1, $n=150$ ) is characterized by methylation upstream of TSS, high FPKM expression values, open chromatin around the TSS, and association with active histone marks (H3K4me2, H3K4me3, H3K9Ac, H3K27Ac) in E11.5 WT embryos (Fig. 3d-f, Supplementary Data 4). The second group (Group 2, $n=163$ ) is characterized by methylation near the TSS, low FPKM expression values, closed chromatin, and association with repressive histone mark H3K27me3 in E11.5 WT embryos (Fig. 3d-f, Supplementary Data 4). In both Groups 1 and 2 , loss of promoter methylation in E11.5 Dnmt3 $b^{-/-}$embryos does not result in gene expression changes.

The third group (Group 3, $n=34$ ) is characterized by methylation near the TSS, low FPKM expression values and closed chromatin, but no enrichment of chromatin marks in E11.5 WT embryos (Fig. 3d-f, Supplementary Data 4). Loss of promoter methylation in E11.5 Dnmt3 $^{-/-}$embryos results in strong up-regulation of gene expression.

These results suggest that in a subset of promoters not enriched for any of the tested histone modifications, DNA methylation plays critical role as transcriptional inhibitor whereas in genes already expressed or marked by $\mathrm{H} 3 \mathrm{~K} 27 \mathrm{me} 3$ its regulatory role is substantially lessened, and represents only one layer of regulation whose removal in Dnmt3 $b^{-/-}$setting does not affect expression.

Catalytic activity of Dnmt3b is associated with H3K27me3. Our analysis revealed that 4133 DMRs hypomethylated in Dnmt $3 b^{-/-}$embryos are similarly methylated between Dnmt $3 b^{+/+}$and Dnmt3b $b^{C I / C I}$ DNA in E11.5 suggesting that their proper methylation primarily depends on the accessory function (Fig. 4a, Supplementary Data 5). These loci are weakly dependent on Dnmt3b's CA and therefore we term them weak. An example of weak DMR is shown for the Bcl11b locus (Fig. 4b). In contrast, 205 loci remain largely hypomethylated in Dnmt3b $b^{C I / C I}$ embryos, suggesting that their methylation strongly depends on Dnmt3b's CA (Fig. 4a, Supplementary Data 5) and we term them as strong 


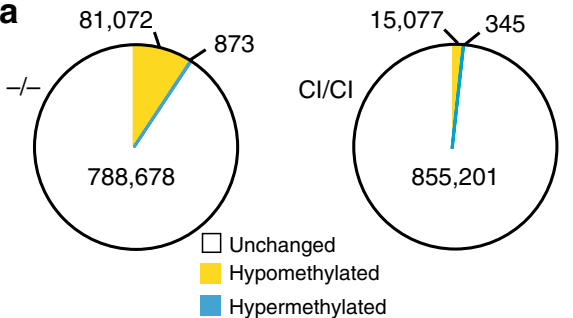

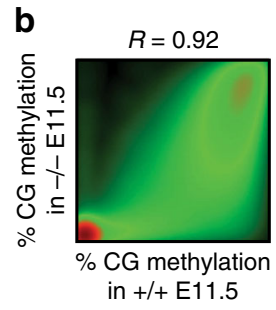

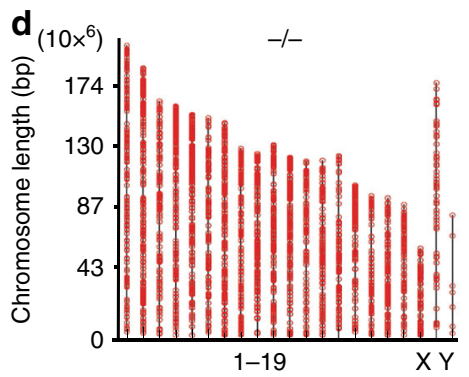

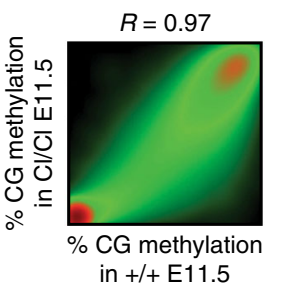

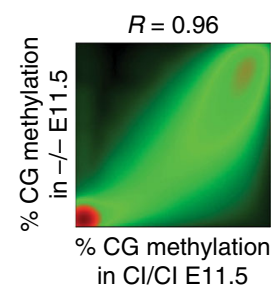

C

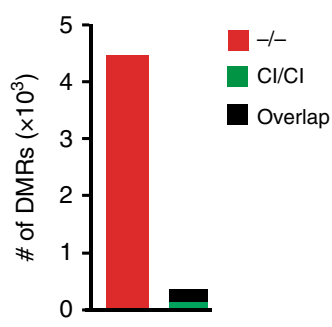

e

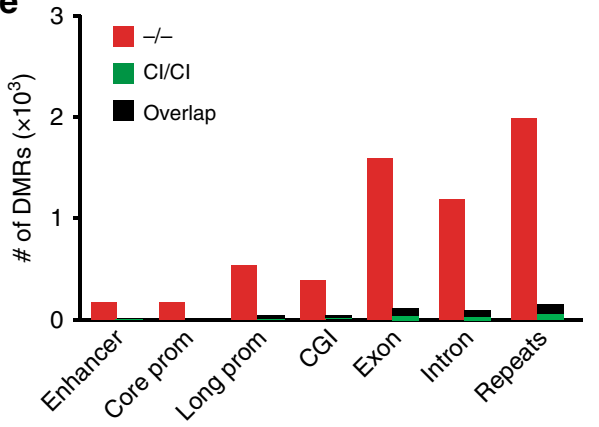

h

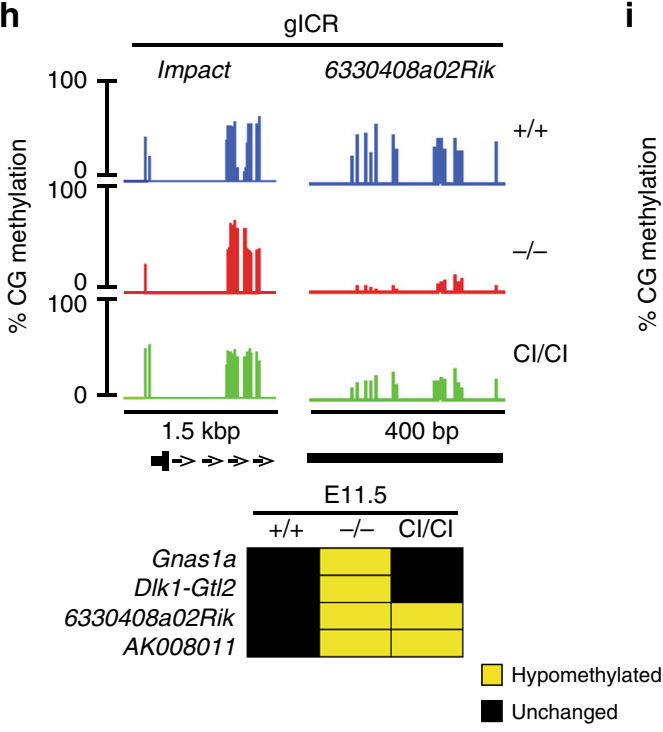

f
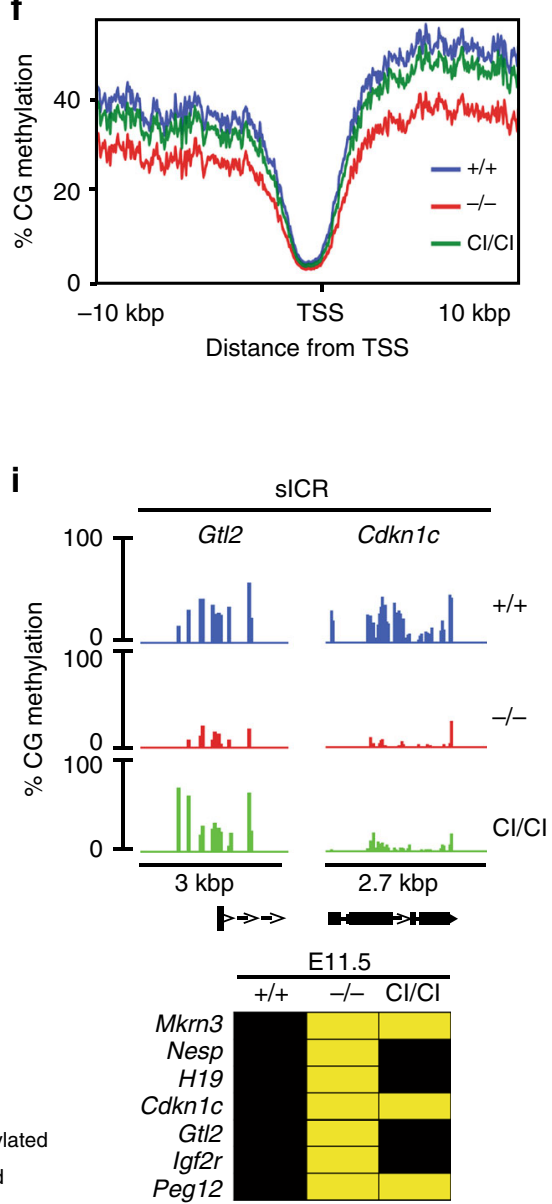

$\mathrm{Cl} / \mathrm{Cl}$

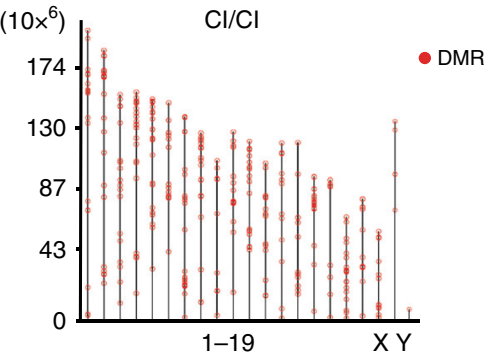

g

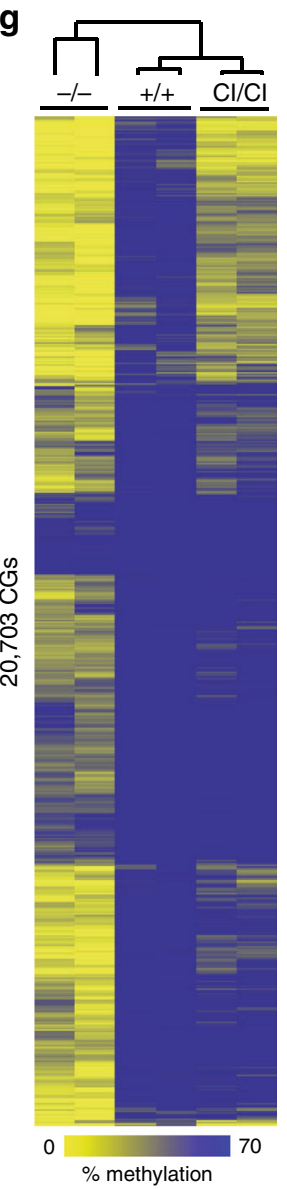

(for example Sycp1; Fig. 4b). After annotating DMRs to genomic features, we found that both groups are relatively equally distributed across the genome with the majority of DMRs found in repetitive elements and gene bodies (Fig. 4c, Supplementary Fig. 10).

By comparing relative enrichments of histone marks in Dnmt3b $b^{+/+}$embryo (ENCODE-ENCSR231EPI, ENCSR215 $\mathrm{ZYV})^{25}$ between genomic areas associated with strong and weak DMRs in Dnmt $3 b^{-/-}$and Dnmt $3 b^{C I / C I}$ embryos, we found that weak DMRs are enriched in activating H3K36me3 when compared to the areas of strong DMRs (Fig. 4d). In contrast, strong DMRs are associated with higher levels of repressive mark $\mathrm{H} 3 \mathrm{~K} 27 \mathrm{me} 3$ relative to weak DMRs (Fig. 4d). No significant differences in other histone marks were observed for weak and strong DMRs on a genome-wide level (Fig. 4d, Supplementary Fig. 11). However, further analysis of genomic elements revealed that the areas of weak DMRs are enriched for H3K36me3 only in exons but not in other parts of the genome. Weak DMRs are also associated with higher levels of $\mathrm{H} 3 \mathrm{~K} 4 \mathrm{me} 2$ and $\mathrm{H} 3 \mathrm{~K} 4 \mathrm{me} 3$ in core promoters and enhancers relative to strong DMRs (Fig. 4e). 
Fig. 2 Dnmt $3 b^{C l}$ restores deregulated methylation in Dnmt3b-/- embryos. a Total number of differentially methylated CGs in Dnmt3b-/- and Dnmt3bCl/Cl E11.5 embryos relative to Dnmt $3 b^{+/+}$counterparts as analyzed by RRBS ( $n=2$ each). Differential CG methylation is defined as $\geq 30 \%$ change. $\mathbf{b}$ Pairwise

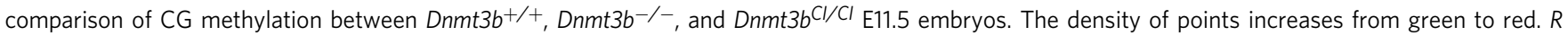
values represent Pearson correlation coefficients. c The number of DMRs ( $\geq 3$ consecutive DMCs in the same direction in the distance $\leq 200$ bp, average DMC methylation change $\geq 30 \%, p<0.05$ ) identified in Dnmt $3 b^{-/-}$and Dnmt3b $b^{C l} C l$ E11.5 embryos relative to Dnmt3b $b^{+/+}$counterparts. Overlapping DMRs between Dnmt $3 b^{-/-}$and Dnmt $3 b^{C l} / C^{C l}$ are marked with black. d Distribution of DMRs on chromosomes identified in Dnmt3b-/- and Dnmt $3 b^{C l} / C^{-}$ E11.5 embryos relative to Dnmt3b+/+ counterparts. Only regions with sufficient coverage are shown. e The number of DMRs associated with enhancers, core promoters ( -300 to $+150 \mathrm{bp}$ relative to TSS), long promoters ( -1500 to $+500 \mathrm{bp}$ ), CGls, exons, introns, and repeats identified in Dnmt $3 b^{-/-}$ and $\mathrm{Dnmt3b} \mathrm{Cl}^{\mathrm{Cl}} \mathrm{E} 11.5$ embryos relative to Dnmt3b+/+ counterparts. $\mathbf{f}$ Distribution of CG methylation in the region from -10 to $+10 \mathrm{kbp}$ around TSS in Dnmt3b $+/+$, Dnmt3b-/-, and Dnmt3b $\mathrm{bl}^{-\mathrm{Cl}}$ E11.5 embryos. $\mathbf{g}$ Hierarchical clustering of CG methylation in E11.5 embryos of indicated genotypes. Heat map shows 20,703 CGs included in all DMRs identified in Dnmt3b-/- and Dnmt3b $\mathrm{Cl} / \mathrm{Cl}$ embryos. $\mathbf{h}$ Percentage of methylation at individual CGs in germline Imprinted Control Region (gICR) Impact (chr18: 12,973,600-12,972,150) and 6330408a02Rik (chr7: 13,261,170-13,261,570) as visualized by IGB software (top). Summary of all hypomethylated gICRs identified in Dnmt3b-/- and Dnmt3b Cl/Cl embryos (bottom). i Percentage of methylation at individual CGs in somatic Imprinted Control Region (sICR) GtI2 (chr12: 109,539,000-109,542,000) and Cdkn1c (chr7:143,459,000-143,461,700) as visualized by IGB software (top). Summary of all hypomethylated sICRs identified in Dnmt $3 b^{-/-}$and Dnmt $3 b^{C l} / C l$ embryos (bottom). Source data are provided as a Source Data file

Strong DMRs are enriched in $\mathrm{H} 3 \mathrm{~K} 27 \mathrm{me} 3$ in exons and long promoters. These associations were not limited to fetal liver but were seen also in neural tube (Supplementary Fig. 12).

Altogether, our data suggest that different mechanism may cover methylation of strong versus weak DMRs. For weak, Dnmt3b may serve as an accessory protein, whereas for strong its $\mathrm{CA}$ is irreplaceable.

Our results suggest different mode of action for Dnmt3b depending on whether catalytic or accessory activity is required. $\mathrm{CA}$ is favored by the presence of repressive $\mathrm{H} 3 \mathrm{~K} 27 \mathrm{me} 3$ whereas accessory activity is promoted by the presence of activating marks such as H3K36me3 in exons and H3K4me2 and H3K4me3 in regulatory elements.

Dnmt3b contributes to de novo methylation at E11.5. After a wave of de novo methylation in early epiblast, the levels remain stable at least to E10.5, but whether any changes occur at E11.5 is unclear $^{13}$. To address this, we first compared methylation of E8.5 and E10.5 WT embryos obtained previously ${ }^{13}$, using readouts of $>5 \times 10^{5}$ CGs (covered at $\geq 15 x$ depth in all samples) and found no differences consistently with previous report ${ }^{13}$ (Fig. 5a). In contrast, we observed substantial gains and losses in CGs methylation between E10.5 and E11.5 WT embryos affecting various genomic elements (Fig. 5a-c, Supplementary Fig. 13). Methylation gains were partially suppressed in E11.5 Dnmt3b $b^{-/-}$embryos, but mostly restored in Dnmt $3 b^{C I / C I}$ embryos suggesting that Dnmt3b plays de novo role in methylation between E10.5 and E11.5 (Fig. 5a-c, Supplementary Fig. 13). For example, the Ppp1r2 locus is not methylated at E8.5, minimally at E10.5 and gains substantial methylation at E11.5 in WT but not in Dnmt3b-/- embryos (Fig. 5d). Partial methylation is observed in $D n m t 3 b^{C I / C I}$ embryos although not to WT levels (Fig. 5d). Gain of methylation was observed even between Dnmt3b-/- E8.5 and E11.5, suggesting involvement of other Dnmts in de novo methylation (Fig. 5a).

Further analysis revealed 133 hyper- and 196 hypomethylated DMRs between E10.5 and E11.5 Dnmt3b $b^{+/+}$embryos (Fig. 5e, f). Out of these, gains in methylation of 18 DMRs in Dnmt $3 b^{+/+}$ embryos are likely dependent on Dnmt3b, as they are not present in Dnmt3b $b^{-/-}$embryos (Fig. 5e, f). This dependence stems from an accessory rather than CA of Dnmt3b as the majority of gains are present also in Dnmt3b $b^{C I / C I}$ (Fig. 5e, f). Effects of methylation gains on gene expression were modest and were limited to $3 / 10 \mathrm{de}$ novo methylated core promoters including $R b m 44, Z f p 995$, and Sfrfbp1 (Fig. 5g).

Despite prevalent increases in methylation, we identified more hypo- than hypermethylated DMRs affecting various genomic elements (Fig. 5e, Supplementary Fig. 14), suggesting that methylation gains are spread across genome while losses are localized to discrete regions of various genomic elements. Loss of methylation does not seem to affect gene expression and its relevance is unclear.

Altogether, our data identified changes in global methylation reflected by gains and losses in a subset of genomic elements and with moderate effects on transcription of few genes.

Dnmt3b regulates major developmental pathways. The majority of Dnmt3b-/- embryos die by age E12.5, suggesting that deregulated molecular events responsible for lethality accumulate by this stage of embryogenesis. To identify these events, we next utilized data obtained on gene expression profiles in Dnmt3b $b^{+/+}$, Dnmt $3 b^{-/-}$, and Dnmt3b $\mathrm{CI} / \mathrm{CI}$ embryos at day E11.5. Ingenuity pathway analysis (IPA) using 253 overexpressed and 819 underexpressed genes $(\mathrm{FC} \geq 2)$ in $D n m t 3 b^{-/-}$embryos relative to WT revealed multiple categories linked to embryogenesis.

For example, under the category Physiological system development and function, TOP five subcategories included Nervous system development and function, Tissue development, Skeletal and muscular system development and function, Organismal development, and Embryonic development underlining their link to development (Fig. 6a). Analysis of downregulated genes also identified deregulation of developmental pathways, such as Development of head, Formation of muscle, and Development of sensory organ (Supplementary Fig. 15).

Since Dnmt3b is a repressor, we reasoned that de-repressed rather than downregulated genes drive lethality in Dnmt3b-/embryos. Indeed, a deeper analysis of 28 genes specifically upregulated in the subcategory Embryonic development revealed that these genes were involved in Wnt signaling (Wnt9b, Frzb), FGF signaling (Fgf8), Hedgehog signaling (Shh), Angiogenesis (Frzb, Fos), and 553 pathway (Cdkn1a) (Fig. 6b). The importance of Wnt signaling was further highlighted by Gene Ontology analysis that identified canonical as well as non-canonical signaling pathways as being activated (Fig. 6c, Supplementary Fig. 16).

Altogether, these data suggested that Dnmt3b may be directly involved in regulation of major developmental pathways including Wnt and Hedgehog signaling.

Dnmt3b is a negative regulator of Wnt and hedgehog signaling. Analysis of deregulated expression in Dnmt3b-/- embryos suggested that up-regulation of Wnt $9 \mathrm{~b}$ might be involved in their lethality. Next, we asked if any methylation changes are associated with regulatory elements including promoters, gene bodies, and enhancers that would explain elevated expression. This analysis 
a

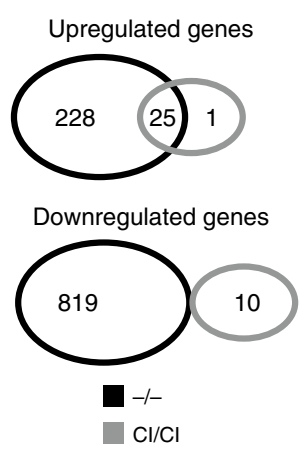

$\mathrm{Cl} / \mathrm{Cl}$ b

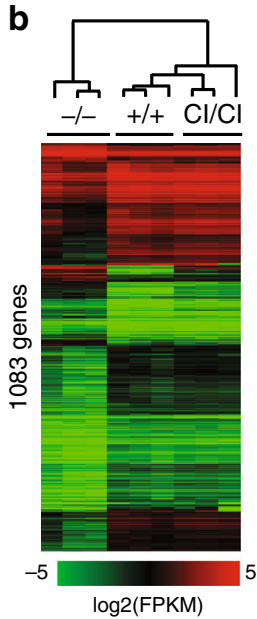

C

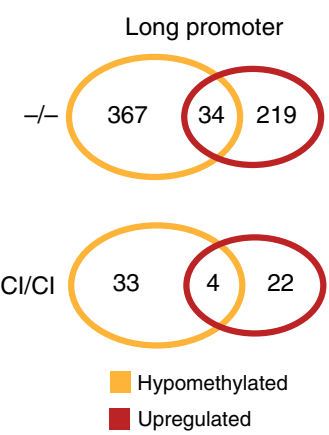

d

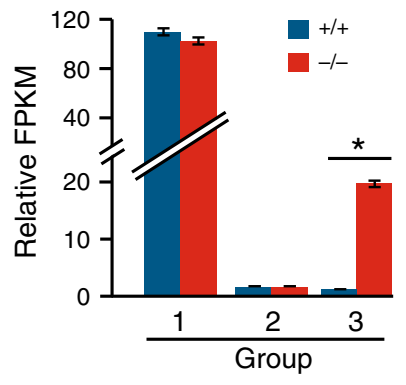

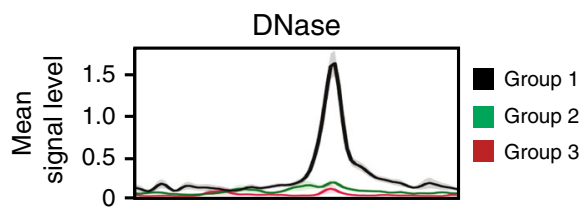

e
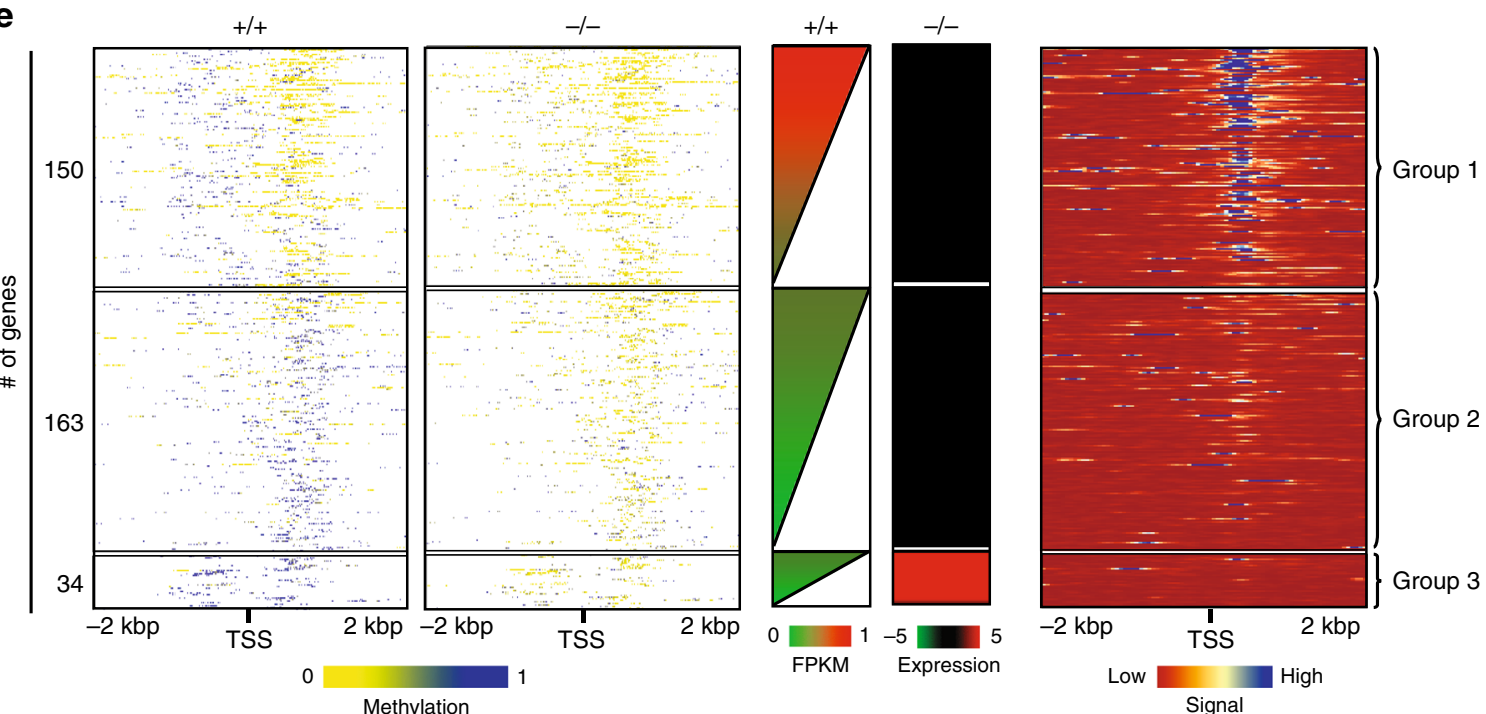

f
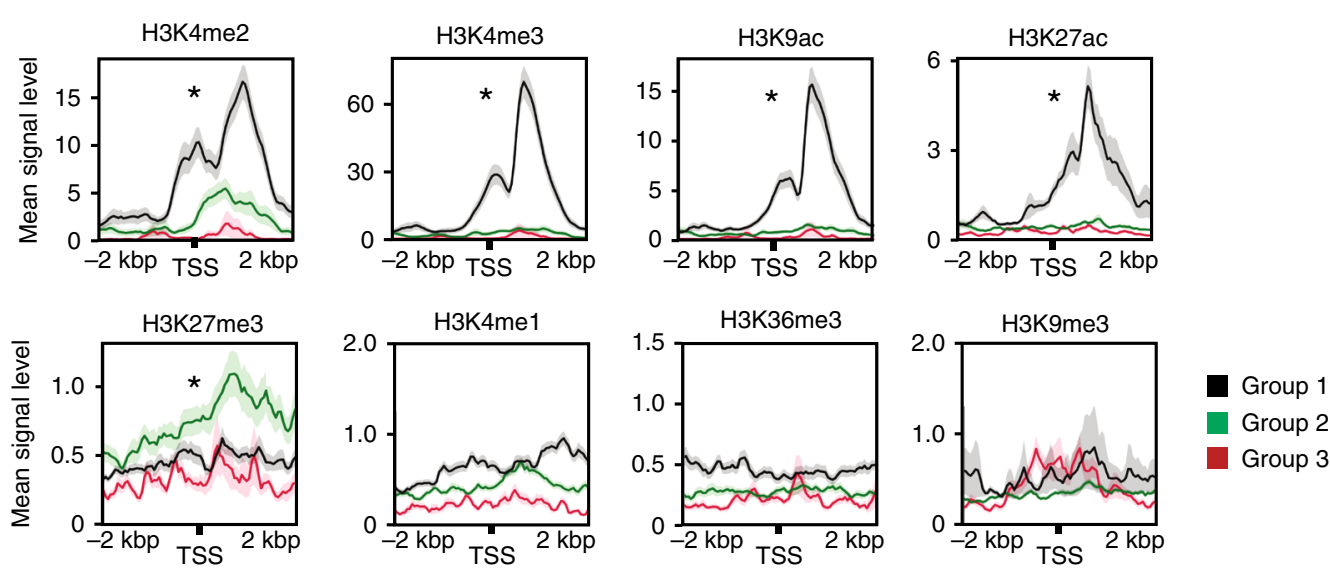

revealed no measurable changes in $22.5 \mathrm{kbp}$ region harboring Wnt9b gene or its putative enhancers among Dnmt $3 b^{+/+}$, Dnmt3b $b^{-/-}$, and Dnmt3b $b^{C I / C I}$ embryos at E11.5 (Fig. 7a, Supplementary Fig. 17). This suggested that $W n t 9 b$ may be regulated by Dnmt3b activities that are not directly linked to catalytic or accessory activity but rather repressive functions or, alternatively, by secondary events involved in embryonic death.
The expression of Wnt9b was significantly increased in E11.5 Dnmt $3 b^{-1-}$ embryos both at RNA and protein level consistently with RNA-seq data (Fig. 7b, c). This up-regulation was not observed in Dnmt $3 b^{C I / C I}$ embryos suggesting that presence of Dnmt $3 b^{C I}$ is sufficient for its normal physiological levels. Upregulation of Wnt9b was more pronounced in fetal brain (Fig. 7d) and was also detectable in Dnmt3b-/- E10.5 embryo 
Fig. 3 Absence of histone modifications is associated with methylation-dependent de-repression. a Weighted Venn diagrams displaying number of differentially expressed genes detected in Dnmt $3 b^{-/-}$and $D n m t 3 b^{C l / C l}$ E11.5 embryos relative to Dnmt3b $b^{+/+}$counterparts. $p<0.001$ by hypergeometric test. b Heat maps of gene subset differentially expressed in embryos of indicated genotypes at E11.5 presented as log2(FPKM) values. Selection of 1083 genes was done by comparing expression values between Dnmt3b-/- and Dnmt3b+/+ embryos $(n=3)$ (FC $\geq 2 ; p<0.05$ by DESeq). c Weighted Venn diagrams displaying overlaps between genes with hypomethylated DMRs and increased expression in Dnmt3b-/- and Dnmt $3 b^{C l / C l}$ embryos relative to Dnmt $3 b^{+/}+$ counterparts; $p<0.001$ by hypergeometric test. $\mathbf{d}$ Relative FPKM values \pm SEM of genes with hypomethylated DMRs in long promoters with high FPKM in Dnmt3 $b^{+/+}$and unchanged in Dnmt3b $b^{-/-}$E11.5 embryos (Group 1, $n=150$ ); low FPKM in Dnmt3b+/+ embryos and unchanged in Dnmt3b $/-$ E11.5 embryos (Group 2, $n=163$ ); and low FPKM in Dnmt3b $+/+$ embryos and increased expression in Dnmt3b-/- (Group 3, $n=34$ ). Data were normalized to mean FPKM values in Group 3 in Dnmt3b+/+ embryos. ${ }^{*}(p<0.01)$. Only genes with $>2$-fold expression increase were included. e Heat map summary of association of methylation and expression levels in Dnmt3b+/+ and Dnmt3b-/- embryos in a region from -2 to +2 kbp around TSS of genes assigned to Groups 1-3. First two panels presents methylation levels of $D n m t 3 b^{+/+}$and Dnmt $3 b^{-/-}$embryos, third panel-FPKM values of Dnmt $3 b^{+/+}$embryos, fourth-change in expression in Dnmt3b-/- relative to Dnmt3b $b^{+/+}$embryos, fifth panel presents results of DNAse I sensitivity assay (ENCODE); plot above shows mean signal \pm SEM for groups 1,2 , and 3 . Genes were sorted based on FPKM values in $+/+$ embryos. $\mathbf{f}$ Plots showing histone marks occupancy signals \pm SEM in region from -2 to $+2 \mathrm{kbp}$ around TSS of genes in groups 1,2 , and 3 ; ${ }^{\star} p<0.05$ by two-tailed Student's $t$-test. SEM values are presented as shading around mean value line. Source data are provided as a Source Data file

(Supplementary Fig. 18), further highlighting its possible role in embryonic death of Dnmt $3 b^{-/-}$mice. Consistently, $\beta$-catenin signaling signature was highly enriched in global gene expression data obtained from Dnmt3b-l- embryos (Fig. 7e). Because Wnt9b was previously found to induce the expression of Fgf8 by binding of $\beta$-catenin to the transcription factor $\mathrm{TCF}^{26}$, we next analyzed expression of this genes specifically in E11.5 Dnmt3b ${ }^{-/-}$ embryos and found elevated levels (Fig. 7f). In addition, we observed negative enrichment of genes involved in developmental processes (Fig. 7g, Supplementary Fig. 19). This is consistent with previous findings ${ }^{27}$ that elevated Fgf8 inhibits a broad range of differentiation pathways including osteogenesis, chondrogenesis, neurogenesis, and myogenesis up to at least E14.5.

Because deregulated expression could be a consequence of impaired development, we next utilized Dnmt $3 b^{-1-}$ mouse lymphoma cells ${ }^{21}$ to test directly the effects of Dnmt3b on Wnt9b and Fgf8 expression. Importantly, exogenous expression of both Dnmt $3 \mathrm{~b}^{\mathrm{WT}}$ and Dnmt $3 \mathrm{~b}^{\mathrm{CI}}$ repressed Wnt $9 \mathrm{~b}$ and $\mathrm{Fg} 8$ (Fig. 7h, i), suggesting that Dnmt3b regulates these genes independently of CA. We next used chromatin immunoprecipitation (ChIP) assays to determine whether Dnmt3b could directly regulate the expression of $\mathrm{Wnt} 9 \mathrm{~b}$. ChIP assays were performed using Dnmt $3 b^{-/-}$mouse lymphoma cells ${ }^{21}$ overexpressing FLAG-tagged version of Dnmt3b ${ }^{W T}$. DNA immunoprecipitated with anti-FLAG antibody was amplified with primers flanking four regions of $W n t 9 b$ promoter $(-800$ to $+200 \mathrm{bp}$ ) (Fig. $7 \mathrm{j})$. The results from these ChIP assays showed enrichment of Dnmt3b in all tested regions especially in -342 to -127 bp region (Fig. $7 \mathrm{j}$ ). Together, these results suggests that Dnmt3b directly represses Wnt9b independent of its CA.

Because enforced expression of Fgf8 and Wnt9b induces embryonic lethality in mice at E11.5-E15.5 (refs. ${ }^{27-29}$ ), upregulation of these genes likely contributes to lethality in Dnmt $3 b^{-1-}$ embryos at E11.5. The importance of repressor activity of Dnmt3b in mouse embryogenesis may be broader since both Dnmt3b ${ }^{\mathrm{WT}}$ and Dnmt $3 \mathrm{~b}^{\mathrm{CI}}$ also repressed expression of Shh, a key member of the hedgehog signaling pathway (Fig. 7k). This observation is consistent with Shh upregulated expression in Dnmt3b-/- embryos at both E10.5 and E11.5 (Supplementary Fig. 18).

Altogether, our data suggest that Dnmt3b controls a wide range of developmental processes through Wnt9b, Fgf8, and hedgehog signaling pathways by its repressor activity that is crucial to prevent embryonic lethality.

\section{Discussion}

In this study, we show that Dnmt3b is critical for embryogenesis by providing accessory function supporting catalytic activities performed by other Dnmts, as well as by exerting gene repression functions controlling essential developmental pathways. We further show that while its $\mathrm{CA}$ is dispensable for mouse embryogenesis it is irreplaceable for a subset of loci distributed across the genome suggesting double role for Dnmt3b in genome methylation. Finally, we show activities independent of the CA of Dnmt3b are linked to control of major developmental pathways including Wnt and hedgehog signaling. Thus, Dnmt3b is a multifaceted protein with several functions critical in mouse embryogenesis.

Dual roles of Dnmt3b in mediating methylation activities were recently reported in colorectal carcinoma cells in vitro ${ }^{30}$. Catalytically inactive Dnmt3b1 induced de novo methylation especially in gene bodies of actively transcribed genes in singly deficient DNMT1 or DNMT3B cells but not in DNMT3A/B double knockout cells. Such results suggested that Dnmt3b is an accessory protein for Dnmt3a mediated enzymatic activities.

We discovered dual capacity of Dnmt3b in embryogenesis described here by comparing methylation in WT, Dnmt $3 b^{-1-}$ and Dnmt3b $b^{C I / C I}$ E11.5 embryos. Accessory activity of Dnmt3b was manifested by loci hypomethylated in Dnmt $3 b^{-/-}$, but not in Dnmt $3 b^{C I / C I}$ embryos. In contrast, strong dependence on intrinsic Dnmt3b's CA was manifested by loci hypomethylated in both genetic settings relative to controls.

Surprisingly, the accessory function of Dnmt3b seems broader in scope than its catalytic function, as methylated loci associated with such activity represent $95 \%$ of identified DMRs in embryogenesis. Association with different histone marks in the WT setting further highlights possible differences in biology of these regions.

In exons, the targets of accessory activity are enriched in activating H3K36me3 unlike targets of CA. Preferential binding of DNMT3B to regions enriched for $\mathrm{H} 3 \mathrm{~K} 36 \mathrm{me} 3$ modification was previously reported, suggesting that DNA methylation might be guided to chromatin carrying this mark ${ }^{11,31}$. In exonic areas, Dnmt3b accessory function may link H3K36me3 to other methyltransferases such as Dnmt3a or Dnmt1 that mediate DNA methylation. In such scenario Dnmt3b would function similarly as catalytically inactive Dnmt3L that links Dnmt3a/b to unmethylated H3K4 (ref. ${ }^{8}$ ). Consistent with such idea is our observation that Dnmt3L is up-regulated in Dnmt3b-/- but not Dnmt3b $b^{C I / C I}$ embryos at E11.5, perhaps due to attempt to compensate for loss of Dnmt3b's accessory function. Targets of Dnmt3b's accessory function in other parts of the genome are not associated with enrichment in $\mathrm{H} 3 \mathrm{~K} 36 \mathrm{me} 3$ and therefore likely involving different histone marks. This is supported by observation of $\mathrm{H} 3 \mathrm{~K} 4 \mathrm{me} 2$ and $\mathrm{H} 3 \mathrm{~K} 4 \mathrm{me} 3$ enrichments in long promoters and enhancers relative to areas dependent on catalytic activity. 
a

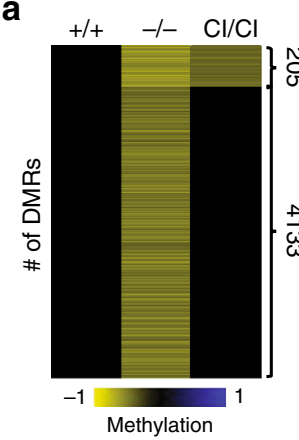

b

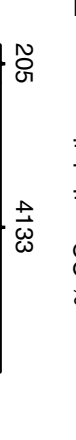
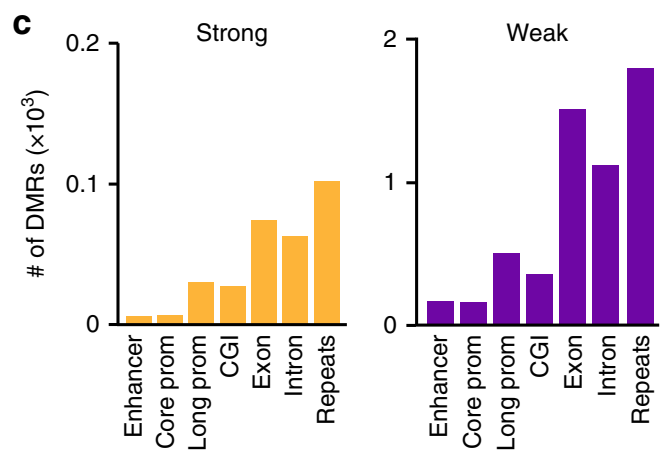

d
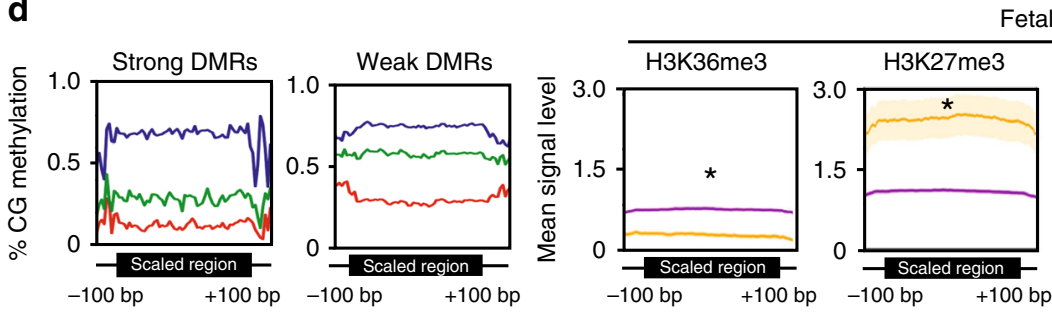

Fetal liver

e

Fetal liver
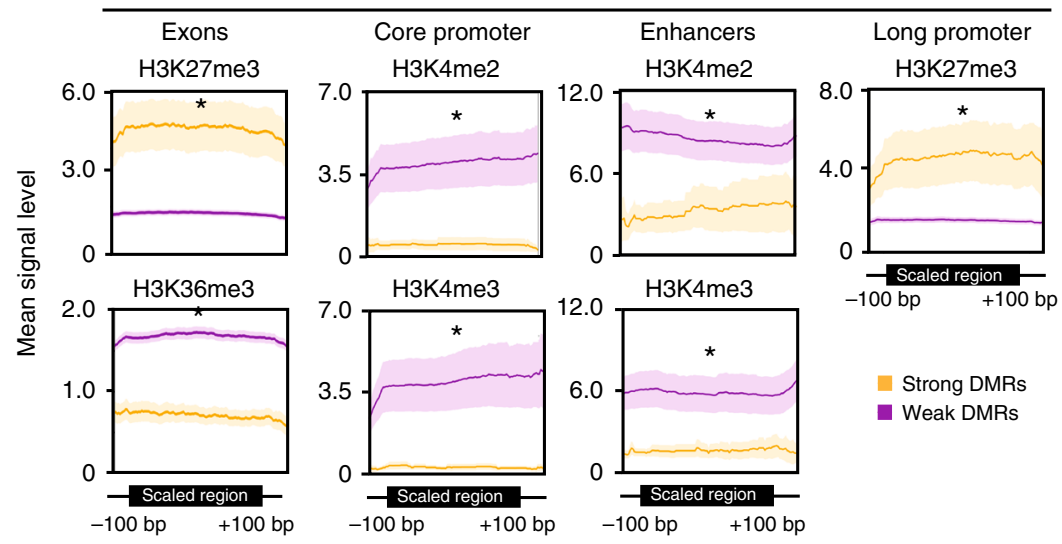

Fig. 4 Association of histone modifications with Dnmt3b-dependent DNA methylation. a Differential requirement of DMRs on catalytic activity of Dnmt3b. Heat map presenting a subset of hypomethylated DMRs $(n=205)$ identified in E11.5 Dnmt3b-/- relative to Dnmt3b $b^{+/+}$depends strongly on catalytic activity of Dnmt3b because their hypomethylation is not rescued to wild-type levels in Dnmt3b $\mathrm{Cl} / \mathrm{Cl}$ E11.5 embryos (termed here strong). In contrast, DMRs hypomethylated in both E11.5 Dnmt3b-/- and $\mathrm{Dnm} t 3 \mathrm{~b}^{\mathrm{Cl} / \mathrm{Cl}}$ relative to wild type depend on accessory rather than catalytic activity of Dnmt3b and are termed here weak $(n=4133)$. b DNA methylation scores obtained for Bcll11b (weak DMR) and Sycp1 (strong DMR) from RRBS analysis of Dnmt3b+/+, Dnmt3b-/- and Dnmt3b Cl/Cl embryos at E11.5 as visualized by IGB. c Distribution of strong and weak DMRs among indicated genomic elements.

d Enrichment of strong DMRs and weak DMRs for chromatin marks in E11.5 Dnmt3b+/+ embryos as detected by analysis of ChIP-seq (ENCODE). Averaged DNA methylation levels \pm SEM for scaled regions of DMRs are shown for strong $(n=205)$ and weak DMRs $(n=4133)$ (first two panels). Mean levels \pm SEM of four histone marks showing significant differences of enrichment between strong and weak DMRs are presented in the right panel. Plots shows profiles for DMRs scaled to same length and surrounding $100 \mathrm{bp}$ unscaled regions. ${ }^{*} p<0.05$ by two-tailed Student's $t$-test. SEM values are presented as shading around mean value line. e Enrichment of strong and weak DMRs in enhancers, exons, core, and long promoters for significantly different chromatin marks in Dnmt3b ${ }^{+/+}$embryos at E11.5 as detected by analysis of ChIP-seq from data obtained from ENCODE. Mean levels of histone marks showing significant differences of enrichment between strong and weak DMRs are presented. Plots shows profiles for DMRs scaled to same length and surrounding 100 bp unscaled regions. ${ }^{\star} p<0.05$ by two-tailed Student's $t$-test. SEM values are presented as shading around mean value line. Source data are provided as a Source Data file

Alternatively, Dnmt3b contributes to stabilization and activation of complexes between DNMT3L and other DNMTs. Indeed, inactive Dnmt3b isoform was shown to stabilize and increase activity of Dnmt3a-Dnmt3L in vitro ${ }^{16}$. While precise mechanism needs to be determined, it is clear that Dnmt3b plays a major accessory role in embryogenesis similar to Dnmt3L.

Previous studies clearly established that Dnmt3L is required for gametic methylation and fertility. Unlike $D n m t 3 b^{C I / C I}$ mice, Dnmt3L $\mathrm{L}^{-/-}$mice show impairment in sperm maturation and oocytes methylation resulting in sterility $8,32,33$. However, this deficiency in vivo does not compromise somatic methylation, as Dnmt3L $L^{-1-}$ mice are viable and display normal methylation patterns in tissues, suggesting that Dnmt3L is not the only accessory protein in embryogenesis ${ }^{8,34,35}$. Additionally, Dnmt3L does not bind directly to H3K36me3 (ref. ${ }^{36}$ ), a major histone modification associated with regions rescued through methylation-independent properties of Dnmt3b. It is therefore possible that one role of Dnmt3b is to provide accessory functions 
a

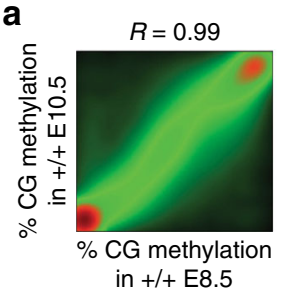

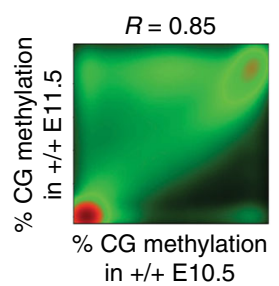

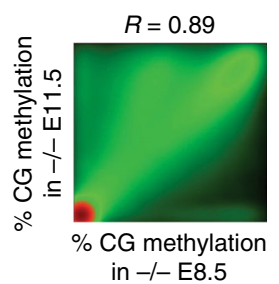

b

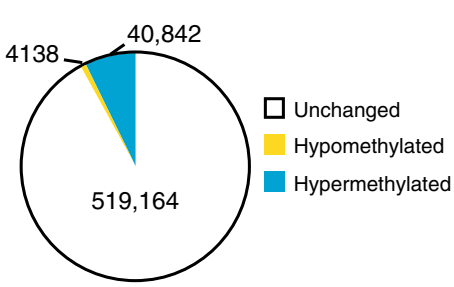

c
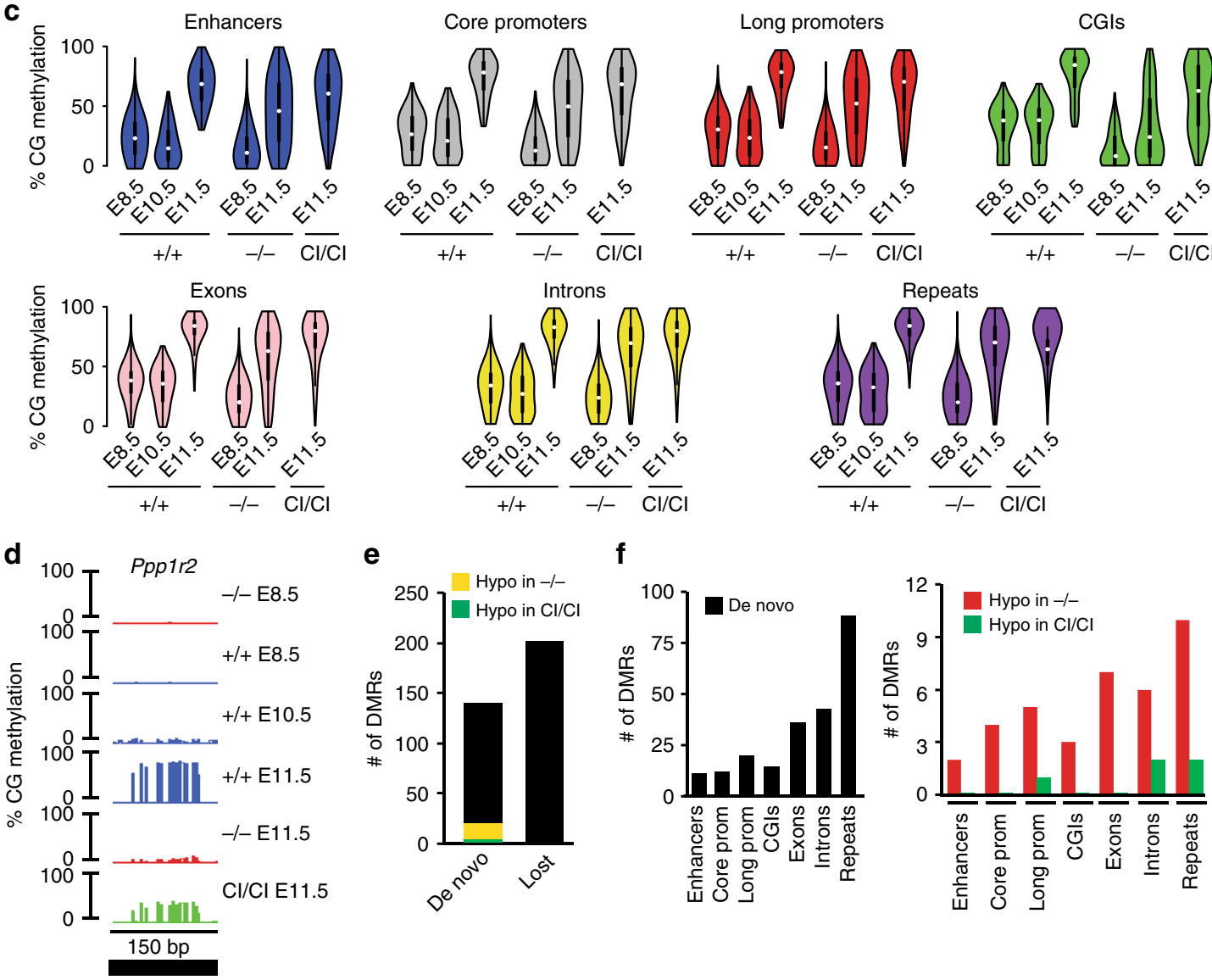

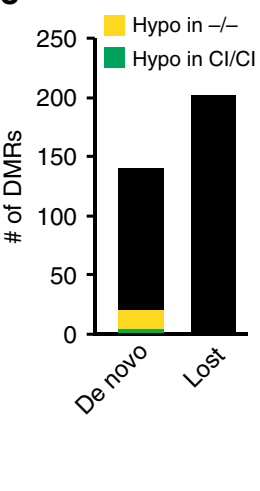

f

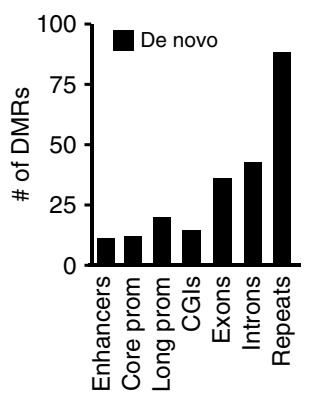

Repeats

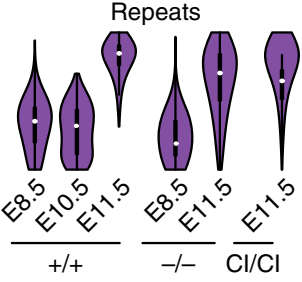

g

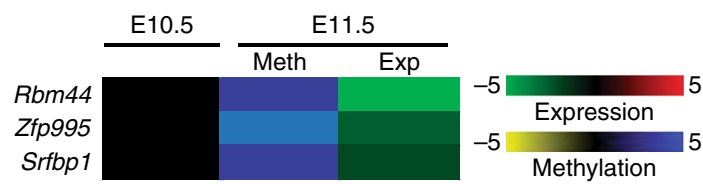

Fig. 5 Methylation changes at E11.5 are partially mediated by Dnmt3b. a Pairwise comparison of CG methylation in Dnmt3b+/+ embryos at E8.5 and E10.5; E10.5 and E11.5 and Dnmt3b-/- embryos at E8.5 and E11.5. The density of points increases from green to red. $R$-values represent Pearson correlation coefficients. b Total number of differentially methylated CGs in Dnmt3b+/+ embryos at E11.5 relative to E10.5 (meth diff. $\geq 30 \%$ ). c Violin plots showing CG methylation in Dnmt3b+/+ embryos at E8.5, E10.5, and E11.5, Dnmt3b-/- embryos at E8.5 and E11.5, and Dnmt3b $\mathrm{Cl} / \mathrm{Cl}$ embryos at E11.5 in indicated genomic elements. d Visualization of single-CpG profiles at de novo methylated locus of Ppp1r2 that remains hypomethylated in Dnmt3b-/- and Dnmt3b Cl/Cl embryos at E11.5. Visualization of single-CpG profiles at Evc2 locus that maintains methylation across E8.5-E11.5 in Dnmt3b+/+ and loses methylation in Dnmt3b $b^{-/-}$. e The total number of hypermethylated (de novo) and hypomethylated (lost) DMRs identified in Dnmt $3 b^{+/+}$embryos at E11.5 relative to E10.5 ( $n=2$ for each timepoint) (methylation diff. $\geq 30 \% ; p(M W U)<0.05$ ). Out of de novo methylated DMRs, those which are hypomethylated in Dnmt3b-/and $\mathrm{Dnmt} 3 b^{\mathrm{Cl} / \mathrm{Cl}}$ relative to Dnmt3b $b^{+/+}$embryos at E11.5 are marked yellow and green, respectively. $\mathbf{f}$ Total number of hypermethylated (de novo) DMRs identified in Dnmt3b $+/+$ embryos at E11.5 relative to E10.5 within indicated genomic elements (left panel). Out of these, DMRs which are hypomethylated in Dnmt $3 b^{-/-}$and Dnmt3b Cl/Cl embryos relative to Dnmt3b $b^{+/+}$at E11.5 are marked red and green, respectively (right panel). $\mathbf{g}$ Heat map of genes de novo methylated within promoter or enhancers and downregulated (FC $\geq 2 ; p<0.05$ by DESeq) in Dnmt3b+/+ embryos at E11.5 relative to E10.5. Source data are provided as a Source Data file

in embryogenesis either throughout embryonic development or after E8.5 when levels of Dnmt3L are decreasing.

Consistent with such idea is the observation that $D n m t 3 b^{-/-}$ embryos die at mid-gestation ${ }^{5,14}$. It remains unanswered which of the Dnmts is recruited by Dnmt3b's accessory function to provide enzymatic activity to targeted loci. While most likely candidate appears to be Dnmt3a, this enzyme is dispensable for embryogenesis $^{5}$. Consequently, the role of Dnmt1 as an enzyme 
a $-\log (p$-value)

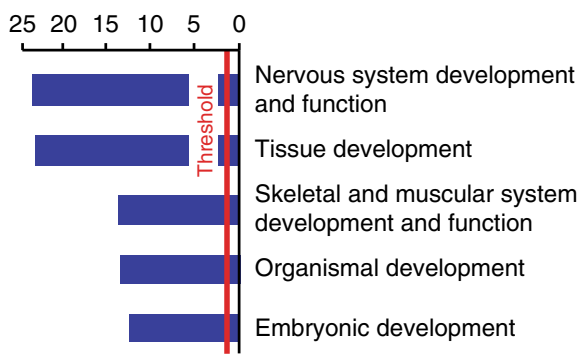

b

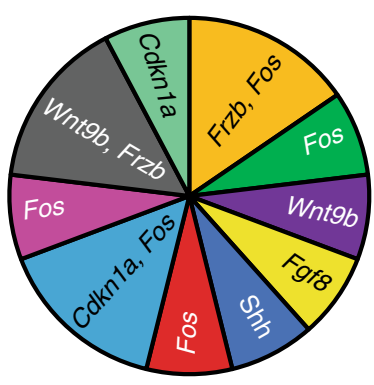

- Angiogenesis

p53 pathway

Wnt signaling pathway

PDGF signaling pathway

Interleukin signaling pathway

Insulin/IGF pathway

Hedgehog signaling pathway

FGF signaling pathway

Cadherin signaling pathway

Apoptosis signaling pathway

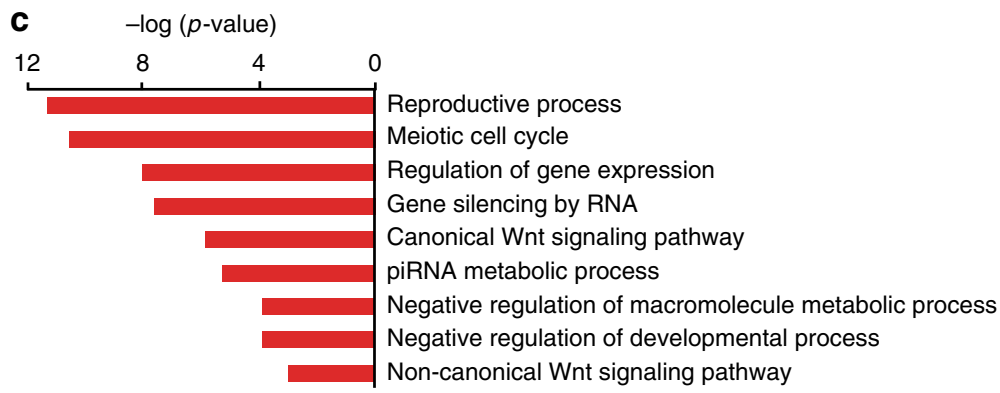

Fig. 6 Dnmt3b controls developmental pathways involved in embryogenesis. a Ingenuity pathway analysis (IPA) of genes differentially expressed (FC $\geq 2$, $p<0.05)$ in E11.5 Dnmt3b-/- embryo. The top subcategories obtained in a category Physiological system development and function are displayed $(p<0.05$ by right-tailed Fisher's exact test). b Panther pathway analysis of upregulated genes (FC $\geq 2, p<0.05$ by DESeq) identified in E11.5 Dnmt3b-/- embryo, which were found to be enriched in IPA analysis in a subcategory Embryonic development. c Gene ontology analysis of up-regulated genes (FC $\geq 2, p<$ 0.05 by DESeq) identified in E11.5 Dnmt3b ${ }^{-/-}$embryo, which were found to be enriched in IPA analysis in a subcategory Embryonic development. Source data are provided as a Source Data file

providing catalytic activity cannot be ruled out, Dnmt1 clearly plays a critical task in embryogenesis as Dnmt1 $1^{-/-}$embryos die at E9.5.

In contrast to accessory activity, targets of CA are associated with higher levels of repressive mark $\mathrm{H} 3 \mathrm{~K} 27 \mathrm{me} 3$ in exons and long promoters. Although DNA methylation and $\mathrm{H} 3 \mathrm{~K} 27 \mathrm{me} 3$ are typically mutually exclusive, it was previously shown that Dnmt $3 \mathrm{~b}$ can engage targets with $\mathrm{H} 3 \mathrm{~K} 27 \mathrm{me} 3$ and methylate the surrounding $\mathrm{DNA}^{2}$. Thus, at least in some genomic areas Dnmt $3 \mathrm{~b}$ executes CA. However, the molecular basis and physiological role of this phenomenon needs further investigation especially because our analysis of histone marks is limited to the wild-type setting.

Another interesting aspect of this study comes from analysis of histone modifications of genes with promoter hypermethylation in WT but hypomethylation in Dnmt $3 b^{-1-}$ E11.5 embryos. Genes associated with open chromatin, active histone marks, and transcription in WT setting did not enhance expression in response to loss of methylation in Dnmt $3 b^{-1-}$ E11.5 embryos. Similarly, loss of methylation did not affect expression of genes associated with closed chromatin and with repressive mark H3K27me3. However, a subset of 34 genes associated with closed chromatin and silencing were strongly up-regulated in Dnmt $3 b^{-/-}$embryos. Interestingly, promoters of these genes were not enriched in any histone mark in Dnmt $3 b^{+/+}$embryos. Thus, in this group, DNA methylation serves as a strong inhibitor of transcription and seems to be a sole determinant whether gene becomes expressed upon loss of DNA methylation. Some of these genes have been previously identified as dependent solely on methylation for their expression, such as Dazl, Piwil2, and Asz1 and being involved in genome defense against transposable elements during the germline cell development $^{37}$. Thus, our data support the idea that certain parts of the genome contain chromatin lacking repressive or activating histone modifications, in which DNA methylation is a key regulatory mechanism of expression ${ }^{37}$.

Our studies also revealed deregulation of pathways potentially involved in embryonic lethality of $D n m t 3 b^{-/-}$mice, including Wnt and hedgehog signaling. We detected up-regulation of Wnt9b that coincides with morphological defects especially observed in the heart of E11.5 Dnmt3b-/- embryos. Importantly, Dnmt3bWT is recruited to Wnt9b promoter and represses expression in vitro, suggesting that Wnt9b is a direct target of Dnmt3b. Similarly, Dnmt $3 b^{C I}$ repressed Wnt9b demonstrating that such effect is independent of MT activity. Whether this repression is mediated through recruitment of HDACs, polycomb complexes, or other mediators remains to be $\operatorname{seen}^{38,39}$.

Wnt signaling is involved in all aspects of embryogenesis and ectopic overexpression of Wnt9b leads to embryonic death at E11.5 to 12.5 primarily due to cardiac insufficiency and hemorrhage $^{28}$. Timing when such up-regulation exerts lethal consequences in embryogenesis and manifestation of similar morphological defects strongly support the idea that upregulation of Wnt9b contributes to lethality of Dnmt3b-1embryos. This is further supported by our observations that Fgf8 is up-regulated in Dnmt3 $b^{-/-}$embryos and ectopic expression of Dnmt3b ${ }^{W T}$ and Dnmt3b ${ }^{C I}$ Dnmt3b represses Fgf8 expression in Dnmt $3 b^{-1-}$ cells in vitro. Fgf8 is activated by Wnt9b and its overexpression in CNC leads to craniofacial defects and embryonic lethality by E14.5 due to disrupted cell differentiation and organogenesis $26,27,29,40$. Hence, up-regulation of Wnt9b and Fgf8 is contributing to embryonic death.

However, increased Wnt signaling may not be the only event involved in lethality of Dnmt3b-/- embryos. We also observed activation of hedgehog signaling through upregulation of Shh in Dnmt $3 b^{-1-}$ embryos. Like for Wnt9b, Shh expression is strongly repressed by overexpression of Dnmt $3 b^{W T}$ and Dnmt3b $b^{C I}$ in 
a

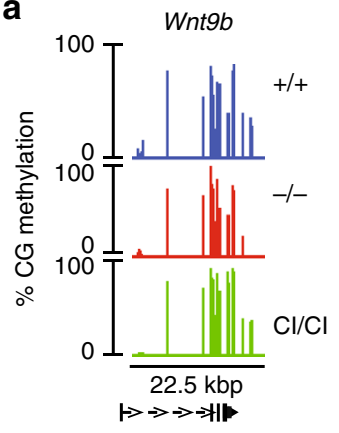

d

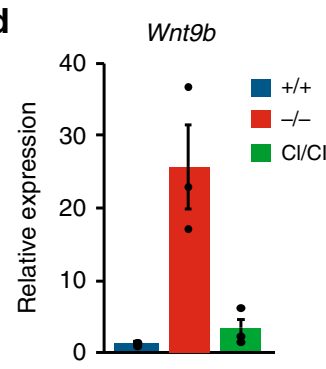

b

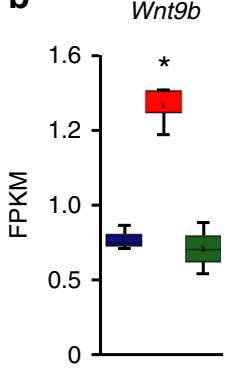

Wnt9b

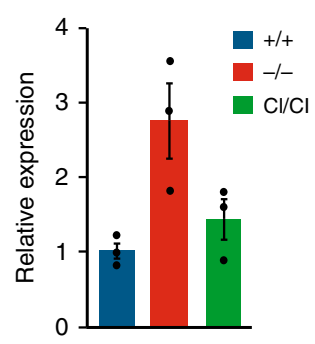

c

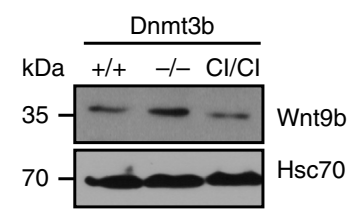

e

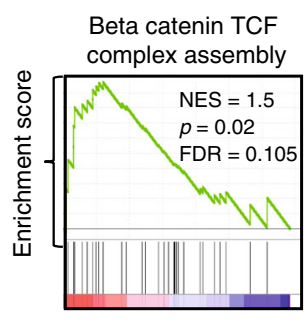

f

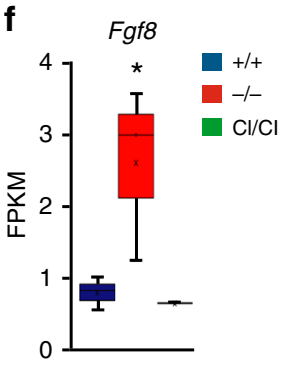

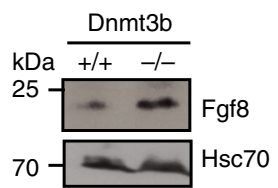

h

g

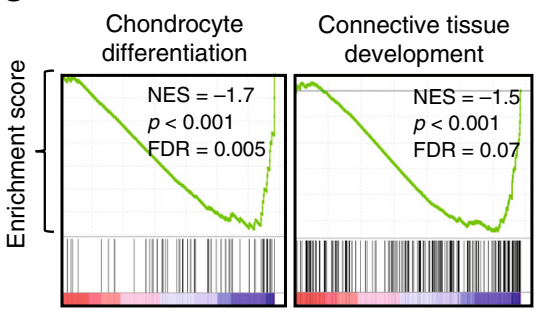

i
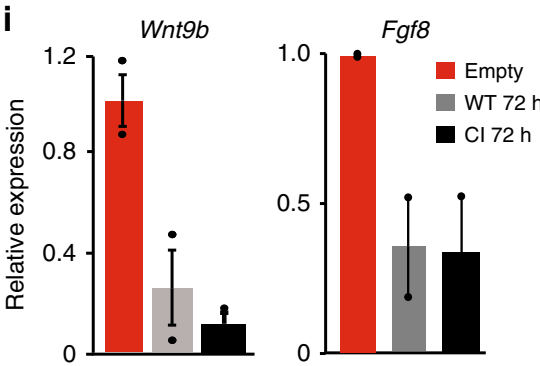

k

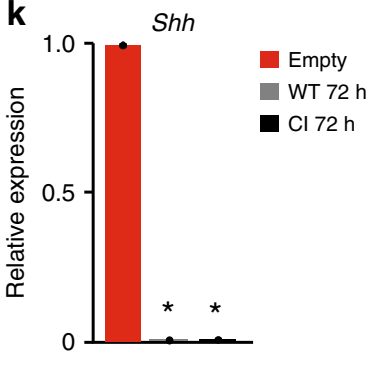

Fig. 7 Dnmt3b represses $W n t 9 b$ and Shh in vivo and in vitro. a Methylation of Wnt9b locus in E11.5 embryos of indicated genotypes determined by RRBS. b Wnt9b expression by RNA-seq $\left(n=3\right.$; ${ }^{*} p<0.05$ by DESeq; left) and real-time qRT-PCR $(n=3$; normalized to Gapdh; right) in E11.5 embryos. Horizontal line represents median, bounds of box-likely range of variation and whiskers-min and max values. c Immunoblot of Wnt9b and Hsc70 levels in E11.5 embryos. d Wnt9b expression by real-time qRT-PCR in fetal brain of E11.5 embryos $(n=3)$, normalized to Gapdh. e GSEA using RNA-seq data shows positive enrichment in Beta-catenin-TCF complex assembly in E11.5 Dnmt3b-/- embryos $(n=3)$. Normalized enrichment scores (NES), false discovery rate (FDR) and $p$ values are shown. $\mathbf{f}$ Left. Fgf8 expression by RNA-seq in Dnmt3b $+/+$, Dnmt3b-/-, and Dnmt3bCl/Cl E11.5 embryos $(n=3),{ }^{\star} p<0.05(\mathrm{DESeq})$. Horizontal line represents median, bounds of box-likely range of variation and whiskers - min and max values. Right. Immunoblot analysis of Fgf8 levels in E11.5 embryos. $\mathbf{g}$ GSEA shows negative enrichment in developmental pathways in E11.5 Dnmt3b-/- embryos relative to Dnmt3b $\mathrm{b}^{+/+}(n=3)$. $\mathbf{h}$ Dnmt3b and Hsc70 expression in mouse Dnmt36-/- T cell lymphoma cells $\left(^{-/-}\right) 72 \mathrm{~h}$ after transduction with lentiviruses expressing control vector (empty),

Dnmt3b WT (3b WT), Dnmt3bCl (3b Cl) as analyzed by immunoblot. i Real-time qRT-PCR analysis of Wnt9b and Fgf8 expression in samples prepared as in h. Average of two independent experiments normalized to $\beta$-actin. $\mathbf{j}$ Left: Schematic of mouse Wnt9b promoter with position of primers (arrows): P1: -763/ -582 bp, P2: $-603 /-411$ bp; P3: $-342 /-127$ bp; P4: $-143 /+36$ bp relative to TSS. Right: Real-time qRT-PCR on DNA immunoprecipitated with antiFLAG antibody from Dnmt3b-/- lymphoma cells expressing FLAG tagged Dnmt3bWT or empty vector. Data are shown as averaged fold enrichment over empty vector $(n=2),{ }^{*} p<0.001$ (two-tailed Student's $t$-test). Assay was performed in triplicates and normalized to the input DNA. $\mathbf{k}$ Shh expression by real-time qRT-PCR in samples described in $\mathbf{h}$. Averaged data of two independent experiments were normalized to $\beta$-actin, ${ }^{*} p<0.001$ (two-tailed Student's $t$-test). Data in figures $\mathbf{b}, \mathbf{d}, \mathbf{i}, \mathbf{j}$, and $\mathbf{k}$ are presented as means \pm SEM. Source data are provided as a Source Data file 
Dnmt3b-/- cells in vitro suggesting involvement of Dnmt3b in its regulation. Shh is a crucial regulator of development including ventral patterning of the neural tube brain and heart ${ }^{41,42}$. Its overexpression in transgenic mice leads to craniorachischisis with major alterations in long bones and death at birth ${ }^{43}$.

Lastly, in this study we also identified E11.5 as a developmental stage at which measurable methylation changes occur. Transition from E8.5 to E10.5 is characterized by stable methylation patterns with minimal changes ${ }^{13}$. In contrast, modest methylation gains and losses occur from E10.5 to E11.5 partially in Dnmt3bdependent manner.

Both gains and losses affected all genomic areas but losses affected more repetitive elements and gene bodies. While modest in scope, gains in promoter methylation are associated with decreased expression of several genes including Rbm44, Zfp 995 and Srfbp1. Thus, E11.5 day of embryogenesis may be marking the beginning of more robust changes accumulated as results of acquisition of tissue-specific methylation patterns.

\section{Methods}

Cell cultures, lentiviruses, and FACS. Lenti-XTM 293 T Cell Line was purchased from Takara (Clontech). Mouse Dnmt $3 \mathrm{~b}^{-/-}$cell line was established as described before $^{21}$. Lentiviral vectors pLV-mDnmt3b-EF1A-mCherry, pLV-mDnmt3bFLAG-EF1A-mCherry, pLV-mDnmt3b (P705V, C706D)-EF1A-mCherry, and pLV-EF1A-mCherry were obtained from VectorBuilder. Cells were maintained in DMEM or RPMI 1640 (Invitrogen) containing 10\% fetal bovine serum. Cell lines were cultured at $37^{\circ} \mathrm{C}$ in a humidified $5 \% \mathrm{CO}_{2}$ atmosphere and were passaged according to recommendations.

To generate lentiviruses, $293 \mathrm{~T}$ cells were seeded in $10-\mathrm{cm}$ tissue culture plate to obtain $\sim 80-90 \%$ confluence and transfected with construct and packaging plasmids psPAX2 and pMD2.G at ratio 1:0.65:0.35 using $70 \mu \mathrm{g}$ of polyethylenimine (PEI) (Polysciences). Virus was collected $48-96 \mathrm{~h}$ post transfection. Transduction was performed as described previously ${ }^{21}$, using either Dnmt $3 b^{-/-}$or Dnmt3a-l-; Dnmt $3 b^{-/-} \mathrm{T}$ cell lymphoma lines ${ }^{20,21}$. Efficiency of transduction was determined by measuring percentage of mCherry + cells by FACS (Supplementary Fig. 20). Transduced cells were harvested $72-96 \mathrm{~h}$ after transduction

For in vitro studies (Fig. 7h, i, k), Dnmt3b ${ }^{\mathrm{WT}}$, Dnmt3b $\mathrm{b}^{\mathrm{CI}}$, and empty vector overexpressing Dnmt $3 b^{-/-}$lymphoma cells were harvested at $72 \mathrm{~h}$ after transduction and used for RNA extraction using standard methods.

For ChIP assay (Fig. 7j), FLAG-tagged Dnmt3b ${ }^{\mathrm{WT}}$-overexpressing Dnmt $3 b^{-/-}$ lymphoma cells were collected at $72 \mathrm{~h}$ after transduction, and cells were immediately processed for ChIP.

For RRBS analysis (Supplementary Fig. 2), Dnmt3b ${ }^{\mathrm{WT}}$, Dnmt3b $\mathrm{b}^{\mathrm{CI}}$, and empty vector overexpressing Dnmt $3 a^{-1-} ;$ Dnmt $3 b^{-/-}$lymphoma cells were harvested $96 \mathrm{~h}$ post transduction and cells were used for DNA extraction using phenol/chloroform extraction and ethanol precipitation.

Mouse studies. Mice harboring conventional knock-in mutations (P656V and C657D) in Dnmt3b coding sequence (Dnmt3b $b^{\mathrm{CI}}$ ) were generated by microinjection of CRISPR/Cas9 and oligo donor in fertilized eggs independently in FVB and C57BL/6 backgrounds. The mutations (CCATGC > GTCGAC) in donor oligo were introduced into exon 19 of Dnmt3b gene (NM_001003961.4) by homologydirected repair. A silent mutation (GTG to GTC) was also introduced upstream to prevent the binding and re-cutting of the sequence after homology-directed repair. Cas9 mRNA, sgRNA, and donor oligo were co-injected into zygotes for knock-in mouse production. The pups were genotyped by PCR, followed by sequence analysis. Positive founders were bred to generate $D n m t 3 b^{+/ C I}$ mice used in these studies. PCR-based genotyping of genomic DNA isolated from the tails was used to confirm genotypes. Sequences of F1, F2, R primers are in Supplementary Data 6. The offspring of two independent mouse lines for each genetic background were analyzed. No phenotypic differences were observed in behavior of founder-specific offspring. All experimental animal procedures were approved by the Institutional Animal Care and Use Committee (IACUC) at the University of Florida under protocol number 201609589 and complied with all relevant ethical regulations for animal testing and research. All mice were housed in a pathogen-free barrier facility at the UF.

All embryos were obtained from naturally mated $D n m t 3 b^{+/-}$and $D n m t 3 b^{+/ C I}$ mice. The morning on which the vaginal plug was observed was designed E0.5 days. Whole embryos were dissected at E10.5, E11.5, or E12.5 and examined under a microscope. Viability of embryos was assessed by detecting the heart beat and blood supply to the yolk sac. Only live embryos were processed for further analysis. Digital images of embryos were captured with a Zeiss Stemi 305 CAM Digital Stereo Zoom Microscope (Carl Zeiss, Inc., Thornwood, NY).

Genomic DNA was prepared by proteinase K digestion followed by phenol/ chloroform extraction and ethanol precipitation. DNA isolated from yolk sac was taken for genotyping. Total RNA was isolated as previously described ${ }^{20}$ from Dnmt $3 b^{+/+} ;$Dnmt $3 b^{-/-}$and Dnmt $3 b^{C I / C I}$ embryos.

To generate conventional Dnmt3b knockout allele, we used approach described previously ${ }^{21,44,45}$ utilizing Dnmt3b $\mathrm{b}^{2 \mathrm{loxP}}$ mice obtained from E. Li (Novartis Institutes for Biomedical Research, Cambridge, Massachusetts, USA). we generated Dnmt3a $a^{-1-} ;$ Dnmt $3 b^{-/-}$cell line previously ${ }^{20}$. Mice were kept in FVB/N genetic background.

Chromatin immunoprecipitation. Mouse Dnmt36-/- lymphoma cells $\left(1 \times 10^{7}\right)$ transduced with empty or Dnmt3b-FLAG expressing vector were crosslinked in $1 \%$ formaldehyde and quenched with $0.125 \mathrm{M}$ glycine. Cells were washed twice with cold PBS and then resuspended in cell lysis buffer $(10 \mathrm{mM}$ Tris- $\mathrm{HCl} \mathrm{pH} 7.5,10 \mathrm{mM}$ $\mathrm{NaCl}, 3 \mathrm{mM} \mathrm{MgCl}_{2}, 0.4 \% \mathrm{NP}-40$ ) to extract nuclei. Nuclei were lysed by incubating in $100 \mu \mathrm{L}$ SDS lysis buffer (1\% SDS, $10 \mathrm{mM}$ EDTA, $50 \mathrm{mM}$ Tris-HCl pH 8.0, protease inhibitors) on ice for $10 \mathrm{~min}$. DNA was sonicated to yield $200-500 \mathrm{bp}$ chromatin fragments, and cleared by centrifugation. The supernatant was diluted in ChIP buffer $(0.01 \%$ SDS, $1 \%$ Triton X-100, 1 mM EDTA, $20 \mathrm{mM}$ Tris-HCl pH 8.0, $150 \mathrm{mM} \mathrm{NaCl}$, and protease inhibitors). The supernatant containing sheared chromatin was precleared by protein G Dynabeads (Invitrogen; 10004D) and the supernatant was incubated with $10 \mu \mathrm{g}$ of anti-FLAG antibody (Sigma; F3165) overnight at $4{ }^{\circ} \mathrm{C}$ with gentle rotation. Protein G Dynabeads were added and the mixture was rotated for $2 \mathrm{~h}$ at $4^{\circ} \mathrm{C}$. DNA was purified by phenol/chloroform/ isoamyl alcohol and subsequent chloroform extractions and ethanol precipitation Isolated DNA was used in RT-qPCR analysis to measure levels of enrichment of Wnt9b promoter region in cells expressing FLAG-Dnmt3b. Primer sequences for qPCR are provided in Supplementary Data 6.

Histology. Formalin-fixed paraffin-embeded sections $(4 \mu \mathrm{m})$ of whole emrbyos were stained with hematoxylin (Sigma, H9627) for $40 \mathrm{~s}$ and with eosin (SigmaAldrich, HT110116) for $30 \mathrm{~s}$. The tissue sections were mounted with Permount mounting medium (Fisher Scientific, SP15-100). All procedures were conducted by Molecular Pathology Core, University of Florida. Images were generated with a Zeiss Axio Imager 2 microscope (Carl Zeiss, Inc., Thornwood, NY).

Western blotting. Western blots were performed as previously described ${ }^{21}$, using the following antibodies: Dnmt1 (ab188453, Abcam; dilution 1:5000), Dnmt3a (SC-20703, Santa Cruz; dilution 1:1000), Dnmt3b (PA1-884, Thermo Fisher; dilution 1:1000), Dnmt3L (SC-393603, Santa Cruz; dilution 1:1000), Fgf8 (MAB323, R\&D Systems; dilution 1:1000), Wnt9b (AF3669, R\&D Systems; dilution 1:1000), Hsc-70 (SC-7298, Santa Cruz; dilution 1:10,000). Uncropped and unprocessed scans of blots are available in Source Data file.

RRBS and bioinformatics analysis. The RRBS libraries were prepared and sequenced at the Medical Genome Facility at the Mayo Clinic, Rochester and ran on an Illumina HiSeq2500 sequencer. Publicly available RRBS data for Dnmt $3 b^{+/+}$ and Dnmt3b $b^{-1-}$ embryos were obtained from GEO (GSE60334) ${ }^{13}$. Quality check, trimming, filtering, and alignment of reads to the reference genome was performed at the ICBR Bioinformatics Core (UF, Florida). The aligned BAM files were uploaded to the Galaxy web platform ${ }^{46}$. Methylation calling was performed with Methyldackel (v 0.3.0.1) using the mm10-CG index (https://github.com/dpryan79/ MethylDackel.git). For methylation analysis in embryos, only CpG sites with a minimum sequencing depth $15 \times$ were included in analysis. For methylation ana-

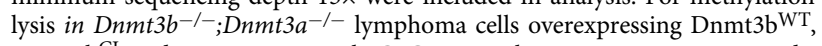
$\mathrm{Dnmt} 3 \mathrm{~b}^{\mathrm{CI}}$, and empty vector, only CpG sites with a minimum sequencing depth of $10 \times$ were included in analysis. Methylation scores were visualized with the Integrated Genome Browser (IGB) ${ }^{47}$. Scatter plots of methylation score were generated in Rstudio v1.1.4.6 using package gplots ${ }^{48}$ and violin plots were generated in BoxplotR $^{49}$. Genome-wide Pearson correlation analysis of $\mathrm{CpG}$ sites was performed using deepTools package multiBigWigsummary and plotCorrelation ${ }^{50}$.

DMRs were determined by Metilene ${ }^{51}$ and defined based on average of minimum three consecutive DMCs with methylation change of $\geq 30$ percent in the same direction with $p$ values $<0.05$ (as determined by MWU test). Maximal base pair cut-off for a distance between consecutive DMCs in DMR was set to 50 or 100

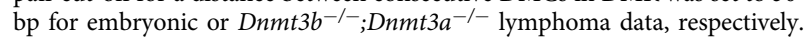

Annotation of methylated CpGs and DMRs to long promoters, core promoters, gene bodies, exons, introns, CGIs, enhancers, and repeats was performed using bedtools intersect. The DMR was retained if the overlap between these elements and DMR was at least $50 \%$ of the length of the DMR. Chromosomal coordinates of TSS, gene bodies, exons, introns, CGIs, and repeats were acquired from the USCS Table browser. Enhancers coordinates identified in forebrain, hindbrain, heart, limb, and hindlimb bud at E11.5 were obtained from Enhancer atlas ${ }^{52}$. Long promoter was defined as $1500 \mathrm{bp}$ upstream to $500 \mathrm{bp}$ downstream of the TSS. Core promoter was defined as $300 \mathrm{bp}$ upstream to $150 \mathrm{bp}$ downstream of the TSS. Gene body was defined as $500 \mathrm{bp}$ downstream of the TSS up to the end of transcript. Hierarchical clustering of $\mathrm{CpG}$ methylation percentages within DMRs was performed using Cluster 3.0 (ref. ${ }^{53}$ ) and heat maps were visualized in Java TreeView 3.0 (ref. ${ }^{54}$ ). DeepTools ${ }^{50}$ was used to display traces and heat maps. 
ChIP-seq data analysis. Publicly available data on chromatin modifications in Dnmt $3 \mathrm{~b}^{+/+}$embryos at E11.5 were obtained from ENCODE (Bing Ren, UCSD lab, ENCSR231EPI, ENCSR215ZYV) ${ }^{25}$. Heat map of DNAse I sensitivity assay and profiles of peak signals were generated using the computeMatrix, plotProfile and plotHeatmap scripts from the deepTools3 package ${ }^{50}$. Plots showing histone enrichment profiles across DMRs scaled to $1000 \mathrm{bp}$ were computed using $10 \mathrm{bp}$ long non-overlaping bins. Flanking un-scaled -100 and +100 bp regions are shown. Plots presenting histone modification profiles for genes with hypomethylated promoters that become up-regulated or retain their expression in Dnmt $3 b^{-1-}$ embryos show unscaled region from -2 to $+2 \mathrm{kbp}$ around TSS.

Combined bisulfite restriction analysis. Combined bisulfite restriction analysis (COBRA) was carried out as described previously ${ }^{55}$. Briefly, bisulfite conversion of genomic DNA was carried out using the Epitect Bisulfite Kit (Qiagen). PCR products were digested with restriction enzymes BstUI and TaqI (NEB). Digested products were then loaded on an $8 \%$ PAGE gel, separated by electrophoresis, and stained by SYBR Gold (Invitrogen).

Mouse bisulfite specific primers are shown in Supplementary Data 6 .

Bisulfite sequencing. Bisulfite conversion of genomic DNA was carried out using the Epitect Bisulfite Kit (Qiagen). PCR fragments from bisulfite treated DNA were cloned using pGem Easy T-cloning kit (Promega). Plasmid DNA isolated from individual clones was sequenced by Sanger sequencing (Genewiz). Mouse bisulfite specific primers are shown in Supplementary Data 6.

Quantification of $\mathbf{5 m d C s}$. Combined liquid chromatography-tandem mass spectrometry with multiple reaction monitoring (LC-MS/MS-MRM) was applied to quantify $5 \mathrm{dmCs}$ levels in DNA extracted from mouse Dnmt $3 b^{+/+} ;$Dnmt $3 b^{-/-}$and Dnmt3b ${ }^{C I / C I}$ E11.5 embryos ${ }^{56}$. DNA hydrolysis was performed by using DNA Degradase Plus (Zymo Research). Briefly, $400 \mathrm{ng}$ of genomic DNA was mixed with $2 \mu \mathrm{L}$ of $10 \times$ DNA Degradase Reaction buffer, $1 \mu \mathrm{L}$ DNA Degradase Plus and water to make a total reaction volume of $20 \mu \mathrm{L}$. Finally, the reaction was inactivated heating to $70^{\circ} \mathrm{C}$ for $20 \mathrm{~min}$. Liquid phase chromatography was performed using gradient elution from a $2.1 \times 100 \mathrm{~mm}$ Hypersil GOLD aQ C18 reversed-phase column with a guard cartridge and an Accela 600 quaternary pump at $250 \mu \mathrm{L} / \mathrm{min}$ at $40^{\circ} \mathrm{C}$. Transition pairs $(258.2 \rightarrow 141.93 \mathrm{~m} / z$ for $5 \mathrm{HmdC}, 242.2 \rightarrow 126.10 \mathrm{~m} / z$ for $5 \mathrm{mdC}$, and $268.1 \rightarrow 152.3 \mathrm{~m} / z$ for $\mathrm{dG}$ ) were monitored on a Thermo Scientific TSQ Vantage Triple Quadrupole mass spectrometer at dwell times of $0.375 \mathrm{~s}$ each and scan widths of $0.002 \mathrm{~m} / z$. The collisional gas pressure was set at $1.2 \mathrm{mTorr}$. The raw data were analyzed with the Xcalibur Qual Browser using ICIS peak detection with following parameters: baseline windows of 30 units for $5 \mathrm{mdC}$ and $5 \mathrm{HmdC}$, windows of 40 units for $\mathrm{dG}$, the minimum signal-to-noise ratio 3.0, and the peak detection set for the highest peak option. Concentrations of $5 \mathrm{mdC}$ were calculated as a percentage of $2^{\prime}$-deoxyguanosine (dG). The calibrated ranges for the samples were $0-25 \%$ for $5 \mathrm{mdC}$ using a fixed $40 \mathrm{pmol}$ amount of $\mathrm{dG}$ as an internal standard. Replicates for the unknown samples were run in triplicate followed by a blank to eliminate carryover into the next unknown run. All the procedures were carried out by Zymo Research Epigenetic Services (Zymo Research Corp).

Real-time qRT-PCR. RNAs were reverse transcribed with the SuperScript III Reverese transcriptase (Thermo Fisher) using oligo(dT) primers. Real-time qRTPCR was performed with the iQ $^{\text {mw }}$ SYBR $^{\circledast}$ Green Supermix (Bio-Rad) on a CFX96 Touch $^{\text {mit }}$ Real-Time PCR Detection System (Bio-Rad). Fast PCR cycling conditions were used $\left(95^{\circ} \mathrm{C}\right.$ for $3 \mathrm{~min}, 40$ cycles $\left(95^{\circ} \mathrm{C}\right.$ for $10 \mathrm{~s}, 58-63.5^{\circ} \mathrm{C}$ for $\left.30 \mathrm{~s}\right)$ ), followed by a dissociation curve analysis. All $\mathrm{qPCR}$ measurements were performed in duplicate reactions and normalized to the expression of three housekeeping genes (Rpl4, $\beta$-actin, Gapdh). In parallel, no-RT controls were amplified to rule out the presence of contaminating genomic DNA. Primer sequences for qPCR are provided in Supplementary Data 6.

RNA-seq. Library generation and sequencing was performed on NovaSeq 6000 platform using paired-end $150 \mathrm{bp}$ runs (Novogene, USA).

Publicly available RNA-seq data for $D n m t 3 b^{+/+}$at E10.5 were obtained from GEO (GSE47033) ${ }^{57}$. Trimmed sequencing data were first aligned to Mus musculus UCSC mm10 reference genome using STAR aligner ${ }^{58}$. RNA-seq data with minimum mapped quality 50 were quantitated using the RNA-seq quantitation pipeline in SeqMonk software (http://www.bioinformatics.babraham.ac.uk/ projects/seqmonk/). DeSeq2 was used to calculate differential expression ${ }^{59}$. For differentially expressed genes, only genes with a fold change $\geq 2$ and a $p$ value $<0.05$ were considered to be significant. Hierarchical clustering was performed in Cluster 3.0 (ref. ${ }^{53}$ ) and heat maps were visualized in Java TreeView 3.0 (ref. ${ }^{54}$ ). Gene ontology enrichment analysis was conducted using the Gorilla software ${ }^{60}$. Pathway enrichment was performed using Panther pathway analysis ${ }^{61,62}$.

Ingenuity pathway analysis. Differentially expressed genes $(\mathrm{FC} \geq 2, p<0.05)$, which were found in Dnmt3b $b^{-/-}$embryos when compared to Dnmt $3 b^{+/+}$embryos and were rescued in Dnmt3b $b^{C I / C I}$ embryos, were analyzed using IPA (Qiagen) ${ }^{63}$. Core analysis was performed to identify top ranking pathways and categories for differentially expressed genes. Top five categories obtained in Physiological system development and function were displayed ( $p<0.05$, for all subcategories).

Gene set enrichment analysis. All FPKM values for Dnmt3b-/-, Dnmt3bCI/CI and Dnmt $3 b^{+/+}$embryos were converted to GCT expression dataset. CLS files were generated using CLSFileCreator (v4) (http://software.broadinstitute.org/cancer/ software/genepattern/modules/docs/ClsFileCreator/4). Hallmark Signature gene sets, Curated gene sets, and GO gene sets were downloaded from Broad Institute's Molecular Signatures Database. Gene Set Enrichment Analysis (v3.0) ${ }^{64,65}$ was used to test the relationship between RNA-seq expression data and the Hallmark Signature gene sets, Curated gene sets and GO gene sets, which were downloaded from Broad Institute's Molecular Signatures Database. Gene sets enriched in less than 15 genes and more than 500 genes were excluded from the analysis. Gene sets with a false discovery rate (FDR) value $<0.25$ and $p<0.05$ after performing 1000 permutations were considered to be significantly enriched.

Statistical analysis. Statistical significance of means \pm SEM were evaluated using the two-tailed Welch's $t$-test. For all statistical analyses $p$ values $<0.05$ were considered significant. The significance between observed and expected genotype representation of embryos was calculated using Chi-squared test. The significance of the overlapping genes was calculated in R version 3.4.3 using the hypergeometric distribution function phyper. Differential histone enrichment between weak and strong DMRs was analyzed by Student's $t$-test or Welch's unequal variances $t$-test with threshold of $p$ value $<0.05$. Clustering was performed on the basis of average linkage hierarchical uncentered clustering of the genes.

Reporting summary. Further information on research design is available in the Nature Research Reporting Summary linked to this article.

\section{Data availability}

The RRBS and RNA-seq data are deposited at the NCBI Gene Expression Omnibus database $^{66}$ under the number GSE132952. All other relevant data supporting the key findings of this study are available within the article and its Supplementary Information files or from the corresponding author upon reasonable request. The source data underlying Figs. le, f, i, j, 3d, 7b, c, d, f, h, i, j, k are provided as a Source Data file. A reporting summary for this article is available as a Supplementary Information file.

Received: 5 April 2019; Accepted: 4 September 2019; Published online: 26 September 2019

\section{References}

1. Zhang, T., Cooper, S. \& Brockdorff, N. The interplay of histone modifications - writers that read. EMBO Rep. 16, 1467-1481 (2015).

2. Zhang, Y. et al. Targets and genomic constraints of ectopic Dnmt3b expression. eLife 7, e40757 (2018)

3. Yin, Y. et al. Impact of cytosine methylation on DNA binding specificities of human transcription factors. Science 356, eaaj2239 (2017).

4. Neri, F. et al. Intragenic DNA methylation prevents spurious transcription initiation. Nature 543, 72-77 (2017)

5. Okano, M., Bell, D. W., Haber, D. A. \& Li, E. DNA methyltransferases Dnmt3a and Dnmt3b are essential for de novo methylation and mammalian development. Cell 99, 247-257 (1999).

6. Li, E., Bestor, T. H. \& Jaenisch, R. Targeted mutation of the DNA methyltransferase gene results in embryonic lethality. Cell 12, 915-926 (1992).

7. Jones, P. A. \& Liang, G. Rethinking how DNA methylation patterns are maintained. Nat. Rev. Genet. 10, 805-811 (2009).

8. Ooi, S. K. et al. DNMT3L connects unmethylated lysine 4 of histone $\mathrm{H} 3$ to de novo methylation of DNA. Nature 448, 714-717 (2007).

9. Barau, J. et al. The DNA methyltransferase DNMT3C protects male germ cells from transposon activity. Science 354, 909-912 (2016).

10. Bachman, K. E., Rountree, M. R. \& Baylin, S. B. Dnmt3a and Dnmt3b are transcriptional repressors that exhibit unique localization properties to heterochromatin. J. Biol. Chem. 276, 32282-32287 (2001)

11. Baubec, T. et al. Genomic profiling of DNA methyltransferases reveals a role for DNMT3B in genic methylation. Nature 520, 243-247 (2015).

12. Smith, Z. D. et al. A unique regulatory phase of DNA methylation in the early mammalian embryo. Nature 484, 339-344 (2012).

13. Auclair, G., Guibert, S., Bender, A. \& Weber, M. Ontogeny of CpG island methylation and specificity of DNMT3 methyltransferases during embryonic development in the mouse. Genome Biol. 15, 545 (2014).

14. Ueda, Y. et al. Roles for Dnmt3b in mammalian development: a mouse model for the ICF syndrome. Development 133, 1183-1192 (2006).

15. Gopalakrishnan, S. et al. A novel DNMT3B splice variant expressed in tumor and pluripotent cells modulates genomic DNA methylation patterns and displays altered DNA binding. Mol. Cancer Res. 10, 1622-1634 (2009). 
16. Van Emburgh, B. O. \& Robertson, K. D. Modulation of Dnmt3b function in vitro by interactions with Dnmt3L, Dnmt3a and Dnmt3b splice variants. Nucleic Acids Res. 39, 4984-5002 (2011).

17. Hsieh, C. L. In vivo activity of murine de novo methyltransferases, Dnmt3a and Dnmt3b. Mol. Cell. Biol. 19, 8211-8218 (1999).

18. Chen, T., Tsujimoto, N. \& Li, E. The PWWP domain of Dnmt3a and Dnmt3b is required for directing DNA methylation to the major satellite repeats at pericentric heterochromatin. Mol. Cell. Biol. 24, 9048-9058 (2004).

19. $\mathrm{Wu}, \mathrm{H}$. et al. Dnmt3a-dependent nonpromoter DNA methylation facilitates transcription of neurogenic genes. Science 329, 444-448 (2010).

20. Haney, S. L. et al. Methylation-independent repression of Dnmt3b contributes to oncogenic activity of Dnmt3a in mouse MYC-induced T-cell lymphomagenesis. Oncogene 34, 5436-5446 (2015).

21. Hlady, R. A. et al. Loss of Dnmt3b function upregulates the tumor modifier Ment and accelerates mouse lymphomagenesis. J. Clin. Invest. 122, 163-177 (2012).

22. Wang, L. et al. Programming and inheritance of parental DNA methylomes in mammals. Cell 157, 979-991 (2014).

23. MacDonald, W. A. \& Mann, M. R. Epigenetic regulation of genomic imprinting from germ line to preimplantation. Mol. Reprod. Dev. 81, 126-140 (2014).

24. Xie, W. et al. Base-resolution analyses of sequence and parent-of-origin dependent DNA methylation in the mouse genome. Cell 148, 816-831 (2012).

25. ENCODE Project Consortium. An integrated encyclopedia of DNA elements in the human genome. Nature 489, 57-74 (2012)

26. Wang, Y., Song, L. \& Zhou, C. J. The canonical Wnt/ß-catenin signaling pathway regulates Fgf signaling for early facial development. Dev. Biol. 349 , 250-260 (2011).

27. Shao, M. et al. FGF8 signaling sustains progenitor status and multipotency of cranial neural crest-derived mesenchymal cells in vivo and in vitro. J. Mol. Cell Biol. 7, 441-454 (2015).

28. Kiefer, S. M., Robbins, L. \& Rauchman, M. Conditional expression of Wnt9b in Six2-positive cells disrupts stomach and kidney function. PLoS ONE 7 e43098 (2012)

29. Schmidt, L., Taiyab, A., Melvin, V. S., Jones, K. L. \& Williams, T. Increased FGF8 signaling promotes chondrogenic rather than osteogenic development in the embryonic skull. Dis. Model Mech. 11, dmm031526 (2018)

30. Duymich, C. E., Charlet, J., Yang, X., Jones, P. A. \& Liang, G. DNMT3B isoforms without catalytic activity stimulate gene body methylation as accessory proteins in somatic cells. Nat. Commun. 7, 11453 (2016).

31. Yang, X. et al. Gene body methylation can alter gene expression and is a therapeutic target in cancer. Cancer Cell 26, 577-590 (2014).

32. Bourc'his, D. \& Bestor, T. H. Meiotic catastrophe and retrotransposon reactivation in male germ cells lacking Dnmt3L. Nature 431, 96-99 (2004).

33. Bourc'his, D., Xu, G. L., Lin, C. S., Bollman, B. \& Bestor, T. H. Dnmt3L and the establishment of maternal genomic imprints. Science 294, 2536-2539 (2001).

34. Kobayashi, H. et al. Contribution of intragenic DNA methylation in mouse gametic DNA methylomes to establish oocyte-specific heritable marks. PLoS Genet. 8, e1002440 (2012).

35. Schulz, R. et al. The parental non-equivalence of imprinting control region during mammalian development and evolution. PLoS Genet. 6, e1001214 (2010).

36. Rondelet, G., Dal Maso, T., Willems, L. \& Wouters, J. Structural basis for recognition of histone $\mathrm{H} 3 \mathrm{~K} 36 \mathrm{me} 3$ nucleosome by human de novo DNA methyltransferases 3A and 3B. J. Struct. Biol. 194, 357-367 (2016).

37. Hackett, J. A. et al. Promoter DNA methylation couples genome-defence mechanisms to epigenetic reprogramming in the mouse germline. Development 139, 3623-3632 (2012).

38. Geiman, T. M. et al. DNMT3B interacts with hSNF2H chromatin remodeling enzyme, HDACs 1 and 2, and components of the histone methylation system. Biochem. Biophys. Res. Commun. 318, 544-555 (2004).

39. Jin, B. et al. DNMT1 and DNMT3B modulate distinct polycomb-mediated histone modifications in colon cancer. Cancer Res. 69, 7412-7421 (2009).

40. Park, J. S. et al. Six2 and Wnt regulate self-renewal and commitment of nephron progenitors through shared gene regulatory networks. Dev. Cell 23, 637-651 (2012).

41. Litingtung, Y. \& Chiang, C. Control of Shh activity and signaling in the neural tube. Dev. Dyn. 219, 143-154 (2000).

42. Washington Smoak, I. et al. Sonic hedgehog is required for cardiac outflow tract and neural crest cell development. Dev. Biol. 283, 357-372 (2005).

43. Tavella, S. et al. Targeted expression of SHH affects chondrocyte differentiation, growth plate organization, and Sox9 expression. J. Bone Min. Res. 19, 1678-1688 (2004).

44. Haney, S. L. et al. Dnmt3a is a haploinsufficient tumor suppressor in CD8+ peripheral T cell lymphoma. PLoS Genet. 12, e1006334 (2016).
45. Haney, S. L. et al. Promoter hypomethylation and expression is conserved in mouse chronic lymphocytic leukemia induced by decreased or inactivated Dnmt3a. Cell Rep. 15, 1190-1201 (2016).

46. Afgan, E. et al. The Galaxy platform for accessible, reproducible and collaborative biomedical analyses: 2018 update. Nucleic Acids Res. 46, W537-W544 (2018).

47. Affymetrix Integrated Genome Browser (IGB). http://www.affymetrix.com/ partners_programs/programs/developer/tools/download_igb.affx.

48. Warnes, G. R. et al. gplots: various R programming tools for plotting data. R package version 2 (2009).

49. Spitzer, M., Wildenhain, J., Rappsilber, J. \& Tyers, M. BoxPlotR: a web tool for generation of box plots. Nat. Methods 11, 121-122 (2014).

50. Ramírez, F., Dündar, F., Diehl, S., Grüning, B. A. \& Manke, T. deepTools: a flexible platform for exploring deep-sequencing data. Nucleic Acids Res. 42, W187-W191 (2014)

51. Jühling, F. et al. metilene: fast and sensitive calling of differentially methylated regions from bisulfite sequencing data. Genome Res. 26, 256-262 (2016).

52. Gao, T. et al. EnhancerAtlas: a resource for enhancer annotation and analysis in 105 human cell/tissue types. Bioinformatics 32, 3543-3551 (2016).

53. de Hoon, M. J. L., Imoto, S., Nolan, J. \& Miyano, S. Open source clustering software. Bioinformatics 20, 1453-1454 (2004).

54. Saldanha, A. J. Java Treeview-extensible visualization of microarray data. Bioinformatics 20, 3246-3248 (2004).

55. Peters, S. L. et al. Essential role for Dnmt1 in the prevention and maintenance of MYC-induced T cell lymphomas. Mol. Cell. Biol. 33, 4321-4333 (2013).

56. Song, L., James, S. R., Kazim, L. \& Karpf, A. R. Specific method for the determination of genomic DNA methylation by liquid chromatographyelectrospray ionization tandem mass spectrometry. Anal. Chem. 77, 504-510 (2005).

57. Tena, J. J. et al. Comparative epigenomics in distantly related teleost species identifies conserved cis-regulatory nodes active during the vertebrate phylotypic period. Genome Res. 24, 1075-1085 (2014).

58. Dobin, A. et al. STAR: ultrafast universal RNA-seq aligner. Bioinformatics 29 , 15-21 (2013).

59. Anders, S. \& Huber, W. Differential expression analysis for sequence count data. Genome Biol. 11, R106 (2010).

60. Eden, E., Navon, R., Steinfeld, I., Lipson, D. \& Yakhini, Z. “GOrilla: a tool for discovery and visualization of enriched GO terms in ranked gene lists". BMC Bioinformatics 10, 48 (2009).

61. Thomas, P. D. et al. PANTHER: a library of protein families and subfamilies indexed by function. Genome Res. 13, 2129-2141 (2003)

62. Thomas, P. D. et al. Applications for protein sequence-function evolution data: mRNA/protein expression analysis and coding SNP scoring tools. Nucleic Acids Res. 34, W645-W650 (2006).

63. Krämer, A., Green, J., Pollard, J. Jr \& Tugendreich, S. Causal analysis approaches in Ingenuity Pathway Analysis. Bioinformatics 30, 523-530 (2014)

64. Subramanian, A. et al. Gene set enrichment analysis: a knowledge-based approach for interpreting genome-wide expression profiles. Proc. Natl Acad. Sci. USA 102, 15545-15550 (2005).

65. Mootha, V. K. et al. PGC-1alpha-responsive genes involved in oxidative phosphorylation are coordinately downregulated in human diabetes. Nat. Genet. 34, 267-273 (2003).

66. NCBI Gene Expression Omnibus. http://www.ncbi.nlm.nih.gov/geo.

\section{Acknowledgements}

The authors thank John Aris, Ph.D., Department of Anatomy and Cell Biology, UF, for help with analysis of embryonic development. The authors thank the Flow Cytometry and Bioinformatics core facilities of the Interdisciplinary Center for Biotechnology Research at the University of Florida. The authors also thank the Genome Analysis core at Mayo Clinic Cancer Center, Rochester, for performing RRBS. Mice with expressing catalytically inactive Dnmt3b were generated in collaboration with Cyagen Inc. This study was supported by Department of Anatomy and Cell Biology and Cancer Center a the University of Florida start-up funds, NIH/NCI grant 1R01CA188561-01A1 (R.O.). M.X. is supported by NIH grants R00-CA190886, R35-GM128753, and Thomas H. Maren foundation.

\section{Author Contributions}

P.N., K.L., J.O., and R.O. contributed to the data generation and editing, and writing of the manuscript. S.L.H., P.S., M.X., and A.A. contributed to the data generation and editing. O.G. contributed to the data analysis. A.R. contributed to the gene expression and DNA methylation processing. R.H. contributed the DNA methylation data processing. A.N. and M.S. contributed to the study design and editing. R.O. supervised the project. All authors read and approved the final manuscript.

\section{Competing interests}

The authors declare no competing interests. 


\section{Additional information}

Supplementary Information is available for this paper at https://doi.org/10.1038/ s41467-019-12355-7.

Correspondence and requests for materials should be addressed to R.O.

Peer review information Nature Communications thanks the anonymous reviewer(s) for their contribution to the peer review of this work. Peer reviewer reports are available.

Reprints and permission information is available at http://www.nature.com/reprints

Publisher's note Springer Nature remains neutral with regard to jurisdictional claims in published maps and institutional affiliations. (c) (i) Open Access This article is licensed under a Creative Commons Attribution 4.0 International License, which permits use, sharing, adaptation, distribution and reproduction in any medium or format, as long as you give appropriate credit to the original author(s) and the source, provide a link to the Creative Commons license, and indicate if changes were made. The images or other third party material in this article are included in the article's Creative Commons license, unless indicated otherwise in a credit line to the material. If material is not included in the article's Creative Commons license and your intended use is not permitted by statutory regulation or exceeds the permitted use, you will need to obtain permission directly from the copyright holder. To view a copy of this license, visit http://creativecommons.org/ licenses/by/4.0/.

(C) The Author(s) 2019 